\title{
A generic white pupae sex selection phenotype for insect pest control
}

2

Ward $\mathrm{CM}^{1,12}$, Aumann $\mathrm{RA}^{2,12}$, Whitehead $\mathrm{MA}^{3}$, Nikolouli $\mathrm{K}^{4}$, Leveque $\mathrm{G}^{5,6}$, Gouvi $\mathrm{G}^{4,7}$, Fung $E^{8}$, Reiling $\mathrm{SJ}^{5}$, Djambazian $\mathrm{H}^{5}$, Hughes $\mathrm{MA}^{3}$, Whiteford $\mathrm{S}^{3}$, Caceres-Barrios $\mathrm{C}^{4}$, Nguyen TNM $^{1,11}$, Choo $A^{1}$, Crisp $P^{1,8}, \operatorname{Sim} S^{9}$, Geib $S^{9}$, Marec $F^{10}$, Häcker $I^{2}$, Ragoussis $J^{5}$, Darby $A C^{3}$, Bourtzis $\mathrm{K}^{4,{ }^{*}}$, Baxter $\mathrm{SW}^{11,{ }^{*},}$, Schetelig $\mathrm{MF}^{2,{ }^{*}}$

${ }^{1}$ School of Biological Sciences, University of Adelaide, Australia, 5005

2 Justus-Liebig-University Gießen, Institute for Insect Biotechnology, Department of Insect Biotechnology in Plant Protection, Winchesterstr. 2, 35394 Gießen, Germany

${ }^{3}$ Centre for Genomic Research, Institute of Integrative Biology, The Biosciences Building, Crown Street, Liverpool, L69 7ZB, United Kingdom

${ }^{4}$ Insect Pest Control Laboratory, Joint FAO/IAEA Division of Nuclear Techniques in Food and Agriculture, Seibersdorf, A-1400 Vienna, Austria

${ }^{5}$ McGill University Genome Centre, McGill University, Montreal, Quebec, Canada

${ }^{6}$ Canadian Centre for Computational Genomics (C3G), McGill University, Montreal, Quebec, Canada

7 Department of Environmental Engineering, University of Patras, 2 Seferi str., 30100 Agrinio, Greece

${ }^{8}$ South Australian Research and Development Institute, Waite Road, Urrbrae, South Australia 5064

9 USDA-ARS Daniel K. Inouye US Pacific Basin Agricultural Research Center, 64 Nowelo Street, Hilo, Hawaii, 96720, USA

${ }^{10}$ Biology Centre, Czech Academy of Sciences, Institute of Entomology, Branišovská 31, 37005 Č̌eské Budějovice, Czech Republic

11 Bio21 Molecular Science and Biotechnology Institute, School of BioSciences, University of Melbourne, Australia, 3010

${ }^{12}$ Authors contributed equally to the study

* Corresponding authors:

k.bourtzis@iaea.org simon.baxter@unimelb.edu.au marc.schetelig@agrar.uni-giessen.de

\section{Author Contributions}

RA, CMW, CC, PC, SS, SG, IH, JR, ACD, KB, SWB, MFS designed research; CMW, RA, MAW, KN, GG, EF, SR, MAH, CC, TNMN, AC, SS, SG, ACD, KB, SWB, MFS performed research; RA, CMW, HD, GL, FM, JR, KB, SWB, MFS contributed new reagents/analytic tools; CMW, RA, MAW, KN, GL, GG, HD, SW, TNMN, AC, SS, SG, IH, JR, ACD, KB, SWB, MFS analyzed data; RA, CMW, KN, GL, GG, SR, SW, AC, SS, SG, IH, JR, ACD, KB, SWB, MFS wrote the paper. 


\section{Abstract}

40 Mass releases of sterilized male insects, in the frame of sterile insect technique programs, 41 have helped suppress insect pest populations since the 1950s. In the major horticultural pests 42 Bactrocera dorsalis, Ceratitis capitata, and Zeugodacus cucurbitae, a key phenotype white 43 pupae (wp) has been used for decades to selectively remove females before releases, yet the 44 gene responsible remained unknown. Here we use classical and modern genetic approaches 45 to identify and functionally characterize causal $w p^{-}$mutations in these distantly related fruit fly 46 species. We find that the wp phenotype is produced by parallel mutations in a single, 47 conserved gene. CRISPR/Cas9-mediated knockout of the wp gene leads to the rapid 48 generation of novel white pupae strains in C. capitata and B. tryoni. The conserved phenotype 49 and independent nature of the $w p^{-}$mutations suggest that this technique can provide a generic 50 approach to produce sexing strains in other major medical and agricultural insect pests. 
Tephritid species, including the Mediterranean fruit fly (medfly) Ceratitis capitata, the oriental fruit fly Bactrocera dorsalis, the melon fly Zeugodacus cucurbitae and the Queensland fruit fly Bactrocera tryoni, are major agricultural pests worldwide ${ }^{1}$. The sterile insect technique (SIT) is a species-specific and environment-friendly approach to control their populations, which has been successfully applied as a component of area-wide integrated pest management programs $^{2-4}$. The efficacy and cost-effectiveness of these large-scale operational SIT applications has been significantly enhanced by the development and use of genetic sexing strains (GSS) for medfly, B. dorsalis and Z. cucurbitae ${ }^{5,6}$.

A GSS requires two principal components: a selectable marker, which could be phenotypic or conditionally lethal, and the linkage of the wild type allele of this marker to the male sex, ideally as close as possible to the male determining region. In a GSS, males are heterozygous and phenotypically wild type, whilst females are homozygous for the mutant allele thus facilitating sex separation ${ }^{6-8}$. Pupal color was one of the first phenotypic traits exploited as a selectable marker for the construction of GSS. In all three species, brown is the typical pupae color. However, naturally occurring color mutants such as white pupae $(w p)^{9}$ and dark pupae $(d p)^{10}$ have occurred in the field or laboratory stocks. The wp locus was successfully used as a selectable marker to develop GSS for C. capitata, B. dorsalis and Z. cucurbitae ${ }^{6,11,12}$, however, its genetic basis has never been resolved.

Biochemical studies provided evidence that the white pupae phenotype in medfly is due to a defect in the mechanism responsible for the transfer of catecholamines from the hemolymph to the puparial cuticle ${ }^{13}$. In addition, classical genetic studies showed that the wp phenotype is due to a recessive mutation in an autosomal gene located on chromosome 5 of the medfly genome $^{9,14}$. The development of translocation lines combined with deletion and transposition mapping and advanced cytogenetic studies allowed the localization of the gene responsible for the wp phenotype on the right arm of chromosome 5 , at position 59B of the trichogen polytene chromosome map ${ }^{15}$. In the same series of experiments, the wp locus was shown to be tightly linked to a temperature-sensitive lethal (ts/) gene (position 59B-61C), which is the second selectable marker of the VIENNA 7 and VIENNA 8 GSS currently used in all medfly SIT operational programs worldwide ${ }^{7,15}$.

The genetic stability of a GSS is a major challenge, mainly due to recombination phenomena taking place between the selectable marker and the translocation breakpoint. To address this risk, a chromosomal inversion called D53 was induced and integrated into the medfly VIENNA 8 GSS (VIENNA $\left.8^{\text {D53+ }}\right)^{6,8}$. Cytogenetic analysis indicated that the D53 inversion spans a large region of chromosome 5 (50B-59C on trichogen polytene chromosome map) with the wp locus being inside the inversion, close to its right breakpoint ${ }^{6}$. 
87 Extensive genetic and cytogenetic studies facilitated the development of a physical map of 88 the medfly genome ${ }^{8,16}$. The annotated gene set provided opportunities for the identification of 89 genes or loci-associated mutant phenotypes, such as the wp and $t s l$, used for the construction 90 of GSS $^{16,17}$. Salivary gland polytene chromosome maps developed for $C$. capitata, B. dorsalis, 91 Z. cucurbitae, and B. tryoni show that their homologous chromosomes exhibit similar banding 92 patterns. In addition, in situ hybridization analysis of several genes confirmed that there is extensive shared synteny, including the right arm of chromosome 5 where the C. capitata wp 94 gene is localized ${ }^{8}$. Interestingly, two recent studies identified SNPs associated with the wp phenotype in C. capitata and Z. cucurbitae that were also on chromosome $5^{18,19}$.

In the present study, we employed different strategies involving genetics, cytogenetics, genomics, transcriptomics, gene editing and bioinformatics to identify independent natural mutations in a novel gene responsible for pupal coloration in three tephritid species of major agricultural importance, C. capitata, B. dorsalis, and Z. cucurbitae. We then functionally characterized causal mutations within this gene in $C$. capitata and $B$. tryoni resulting in development of new white pupae strains. Due to its conserved nature ${ }^{20}$ and widespread occurrence in many insect species of agricultural and medical importance, we also discuss the potential use of this gene as a generic selectable marker for the construction of GSS for SIT applications. 


\section{Results}

\section{Resolving the $B$. dorsalis wp locus with interspecific introgression.}

The $B$. dorsalis white pupae phenotype was introgressed into $B$. tryoni to generate a strain referred to as the Bactrocera Introgressed Line (BIL, Supplementary Fig. 1). To determine the proportion of $B$. dorsalis genome introgressed into $B I L$, whole genome sequence data from male and female $B$. dorsalis, $B$. tryoni, and $B I L$ individuals were analyzed. Paired end Illumina short read data from single B. oleae males (SRR826808) and females (SRR826807) were used as an outgroup. Single copy orthologs across the genome ( $n=1,846)$ were used to reconstruct the species topology revealing species-specific monophyly (Fig. 1A) consistent with previously published phylogenies ${ }^{21,22}$. Reconstruction also showed monophyly between B. tryoni and BIL across $99.2 \%$ of gene trees suggesting the majority of loci originally introgressed from $B$. dorsalis have been removed during backcrosses.

Genomes were partitioned into $100 \mathrm{~kb}$ windows and pairwise absolute genetic distance $\left(d_{X Y}\right)$ calculated between each species and BIL to estimate admixture. $B$. dorsalis was found to be highly similar to a small proportion of the BIL genome (Fig. 1B; purple), as indicated by $d_{X Y}$ values approaching the median value of $B$. dorsalis vs $B$. tryoni (Fig. 1B; orange).

Two formal tests for introgression were also carried out, the $f$ estimator $\widehat{f_{d}}$ (Fig. 1C) and topology weighting (Fig. 1D). Three distinct local evolutionary histories (Fig. 1D) were tested using $d_{X Y}$ and topology weighting across the $B$. dorsalis wp Quantitative Trait Locus (QTL) i) $B I L$ is closest to $B$. tryoni (Fig. 1D; purple, expected across most of the genome), ii) $B I L$ is closest to $B$. dorsalis (Fig. 1D; orange, expected at the wp-locus), and iii) $B / L$ is closest to $B$. oleae (Fig. 1D; green, a negative control). Across the nuclear genome the species topology was supported in $98.82 \%$ of windows. Both $\widehat{f}_{d}$ and topology weighting confirmed a lack of widespread introgression from $B$. dorsalis into $B I L$ with few $(n=42)$ discordant outlier windows. Genomic windows discordant across all three tests were considered candidate regions for the wp mutation. Four scaffolds accounting for $1.18 \%$ of the $B$. dorsalis genome met these criteria and only two, NW_011876372.1 and NW_011876398.1, showed homozygous introgression consistent with a recessive white pupae phenotype (Supplementary Fig. 2).

To resolve breakpoints within the $B$. dorsalis wp QTL, a $10 \mathrm{~kb}$ windowed analysis across NW_011876398.1 and NW_011876372.1 was performed using $d_{X Y}$ (Fig. 1E), topology weighting (Fig. 1F) and $\widehat{f}_{d}$ (Fig. 1G). The maximum range of the introgressed locus was 4.49 Mb (NW_011876398.1 was 2.9-5.94 Mb and NW_011876372.1 was 0-1.55 Mb) (Fig. 1E-G). The wp QTL was further reduced to a $2.71 \mathrm{Mb}$ region containing 113 annotated protein coding genes through analyzing nucleotide diversity $(\pi)$ among eight pooled $B I L$ genomes (3.8 Mb on NW_011876398.1 to 0.73 Mb on scaffold NW_011876372.1, Supplementary Fig. 2). 

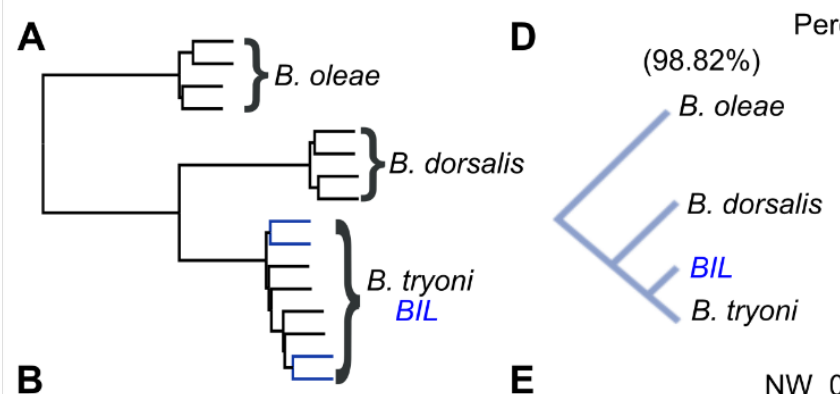

Percentage of genome with topology

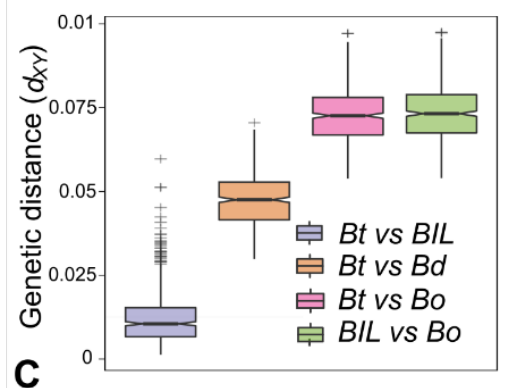

E NW_011876398.1
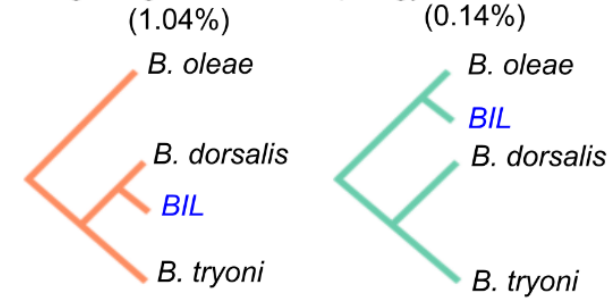

NW_011876372.1
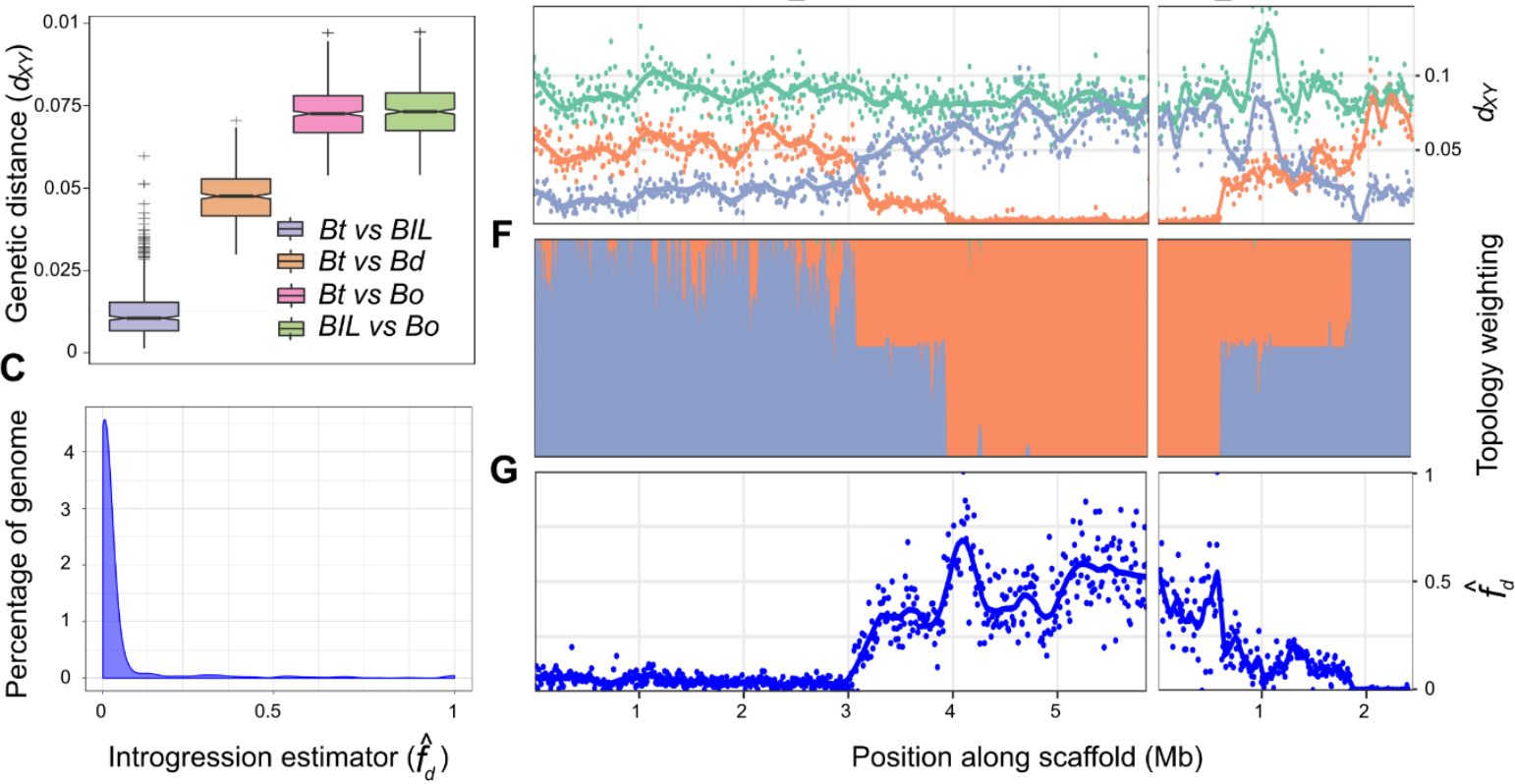

Fig. 1. Characterization of total introgression from $B$. dorsalis into the Bactrocera Introgressed Line and identification of the white pupae locus. (A) Species tree constructed from 1,846 single copy ortholog gene trees for four haplotypes of B. oleae, B. dorsalis, B. tryoni and BIL. Branches corresponding to $B / L$ individuals are shown in blue. All nodes were well supported with posterior probabilities $>0.97$. (B) Nei's absolute genetic distance $\left(d_{X Y}\right)$ calculated for tiled $100 \mathrm{~kb}$ windows across the genome between $B$. tryoni vs $B I L$ (Bt vs BIL); $B$. tryoni vs $B$. dorsalis (Bt vs $B d$ ); $B$. tryoni vs $B$. oleae ( $B t$ vs $B o$ ) and $B I L$ vs $B$. oleae $(B I L$ vs $B o)$. (C) The introgression estimator $\left(\widehat{f_{d}}\right)$ calculated across tiled $100 \mathrm{~kb}$ windows to identify regions of disproportionately shared alleles between $B I L$ and $B$. dorsalis, $\widehat{f_{d}}$ (Bt, BIL, Bd; Bo). (D) The three evolutionary hypothesis/topologies of interest to identify introgressed regions and their representation across the genome: species (purple, 98.82\%), introgression (orange, $1.04 \%$ ) and a negative control tree (green, $0.14 \%$ ). (E) Nei's absolute genetic distance $\left(d_{X Y}\right)$ calculated for tiled $10 \mathrm{~kb}$ windows across the candidate wp locus colors follow the legend in (D). (F) Topology weighting for each topology shown in (D) calculated for $1 \mathrm{~kb}$ tiled local trees across the candidate $w p$ locus. (G) The introgression estimator $\left(\widehat{f_{d}}\right)$ calculated across tiled $10 \mathrm{~kb}$ windows, $\widehat{f_{d}}$ (Bt, BIL, Bd; Bo).

\section{Resolving the C. capitata D53 inversion breakpoints and wp locus with genome} sequencing and in situ hybridization.

Previous cytogenetic studies determined the localization of the gene responsible for the white pupae phenotype on the right arm of chromosome 5, at position 59B of the trichogen polytene chromosome map ${ }^{15}$. The equivalent of position $59 \mathrm{~B}$ is position $76 \mathrm{~B}$ of the salivary gland 
163

76B on the salivary gland polytene chromosome map). Long read sequencing data were generated of the wild type strain Egypt II (EgII), the inversion line D53 and the genetic sexing strain VIENNA 8 (without the inversion; VIENNA $8^{\text {D53-- }}$ ) (Supplementary Table 1) to enable a comparison of the genomes and locate the breakpoints of the D53 inversion, to subsequently narrow down the target region, and to identify wp candidate genes.

Chromosome 5-specific markers ${ }^{16}$ were used to identify the Egll_Ccap3.2.1 scaffold_5 as complete chromosome 5. Candidate D53 breakpoints in Egll scaffold_5 were identified using the alignment of three genome datasets Egll, VIENNA $8^{\text {D53-- }}$, and D53 (see material and methods). The position of the D53 inversion breakpoints was located between 25,455,334 $25,455,433$ within a scaffold gap (left breakpoint), and at 61,880,224 bp in a scaffolded contig (right breakpoint) on Egll chromosome 5 (Ccap3.2.1; accession GCA_902851445). The region containing the causal wp gene was known to be just next to the right breakpoint. Cytogenetic analysis and in situ hybridization using the wild type Egll strain and the D53 inversion line confirmed the overall structure of the inversion, covering the area of $69 \mathrm{C}-76 \mathrm{~B}$ on the salivary gland polytene chromosomes (Fig. 2), as well as the relative position of markers residing inside and outside the breakpoints (Fig. 2 and Supplementary Fig. 3). PCRs using two primer pairs flanking the predicted breakpoints (Supplementary Fig. 4) and subsequent sequencing confirmed the exact sequence of the breakpoints. Using a primer combination specific for the chromosome 5 wild type status confirmed in the WT in Egll flies and VIENNA $7^{\mathrm{D} 53+1}$ GSS males, which are heterozygous for the inversion. Correspondingly, these amplicons were not present in D53 males and females or in VIENNA $7^{\text {D53+|+ }}$ GSS females (all homozygous for the inversion) (Supplementary Fig. 4). Positive signals for the inversion were detected in D53 and VIENNA $7^{\text {D53+ }}$ GSS males and females, but not in WT flies using an inversion-specific primer pair (Supplementary Fig. 4). 

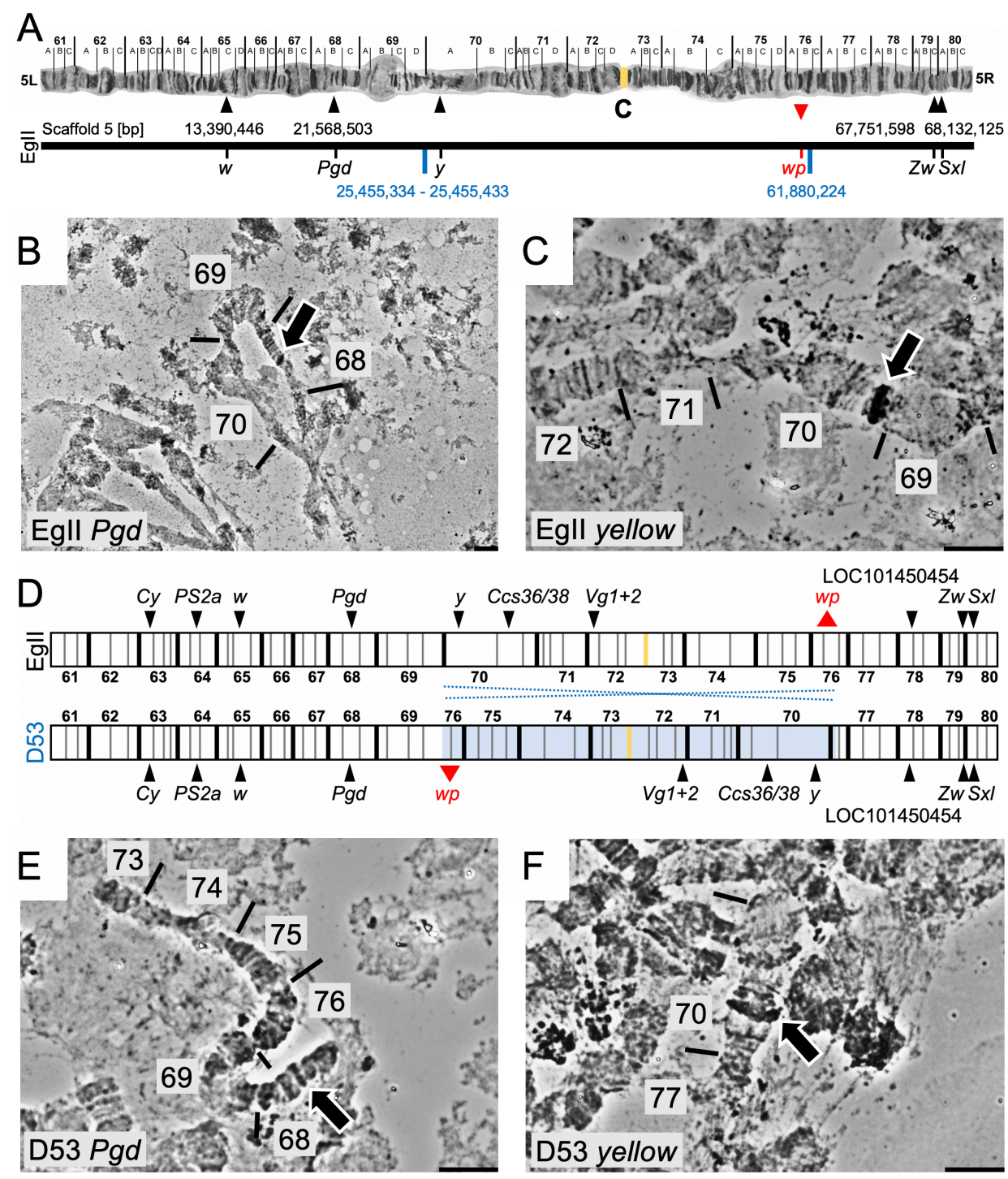

Fig. 2. Genomic positioning of the D53 inversion on chromosome 5 of C. capitata. (A) Chromosome scale assembly of $C$. capitata Egll chromosome 5 . Shown are the positions of in situ mapped genes white $(w)$, 6-phosphogluconate dehydrogenase (Pgd), glucose-6-phosphate 1dehydrogenase $(Z w)$ and sex lethal $(S x I)$, the position of the D53 inversion breakpoints (blue), and the relative position of white pupae (wp) on the polytene chromosome map of chromosome $5^{23}$ and the PacBio-Hi-C Egll scaffold_5, representing the complete chromosome 5 (genome Ccap3.2.1, accession GCA_902851445). The position of the yellow gene ( $y$, LOC101455502) was confirmed on chromosome $570 \mathrm{~A}$ by in situ hybridization, despite its sequence not been included in the scaffold assembly. (D) Schematic illustration of chromosome 5 without (Egll, WT) and with (D53) D53 inversion. The inverted part of chromosome 5 is shown in light blue. Two probes, one inside $(y, 70 \mathrm{~A})$ and one outside $(P g d$, 68B) of the left inversion breakpoint were used to verify the D53 inversion breakpoints by in situ hybridization. WT Egll is shown in (B) and (C), D53 in (E) and (F).

Genome and transcriptome sequencing lead to a single candidate wp gene in $B$. dorsalis, C. capitata, and Z. cucurbitae.

203 Orthologs within the QTL of $B$. dorsalis, C. capitata and scaffolds previously identified to segregate with the wp phenotype in Z. cucurbitae (NW_011863770.1 and NW_011863674.1) ${ }^{18}$ were investigated for high effect mutations under the assumption that a null mutation in a 
conserved gene results in the $w^{-}$phenotype. A single ortholog containing fixed indels absent

207 from wild type strains was identified in each species. White pupae $B$. dorsalis and BIL strains

208 showed a $37 \mathrm{bp}$ frame-shift deletion in the first coding exon of LOC105232189 introducing a

209 premature stop codon $210 \mathrm{bp}$ from the transcription start site (Fig. 3A). Presence of the

210 deletion was confirmed in silico using whole genome resequencing from the wp and wildtype

211 mapped to the reference, and by de novo assembly of Illumina RNAseq data transcripts (Fig.

$2123 \mathrm{~A})$.

213 In C. capitata, wp individuals D53 Nanopore read alignment on Egll showed an

214 independent approximate 8,150 bp insertion into the third exon of LOC101451947 disrupting

215 proper gene transcription 822 bp from the transcription start site (Fig. 3B). The insertion

216 sequence is flanked by identical repeats, suggesting that it may originate from a transposable

217 element insertion. The $C$. capitata mutation was confirmed in silico, as in $B$. dorsalis, using

218 whole genome sequencing and RNAseq data (Fig. 3B).

219 Transcriptome data from the white pupae-based genetic sexing strain of $Z$. cucurbitae 220 revealed a 13 bp deletion in the third exon of LOC105216239 on scaffold NW_011863770.1

221 introducing a premature stop codon (Fig. 3C).

222 The candidate white pupae gene in all three species had a reciprocal best BLAST hit to the 223 putative metabolite transport protein CG14439 in Drosophila melanogaster and contains a 224 Major Facilitator-like superfamily domain (MFS_1, pfam07690), suggesting a general function 225 as a metabolite transport protein. In situ hybridization on polytene chromosomes of $B$. dorsalis, 226 C. capitata and Z. cucurbitae was used to confirm the presence of the wp locus in the same 227 syntenic position on the right arm of chromosome 5 (Fig. 3). Therefore, all three species show 228 a mutation in the same positional orthologous gene likely to be responsible for the phenotype 229 in all three genera. 
A Bactrocera dorsalis
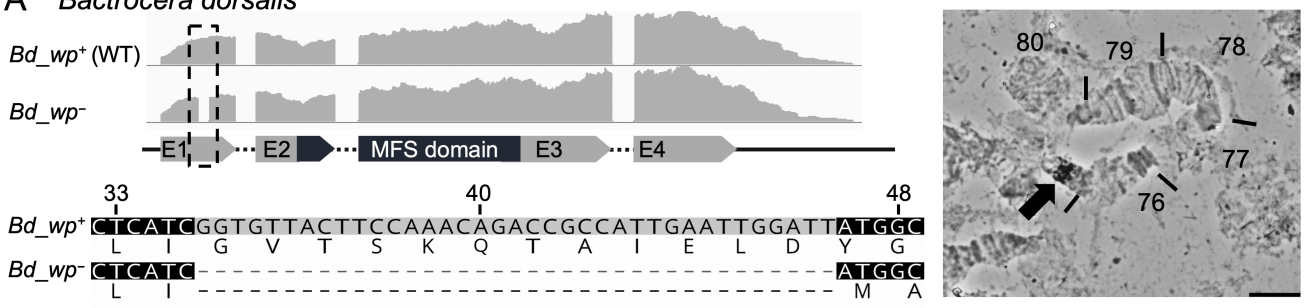

B Ceratitis capitata
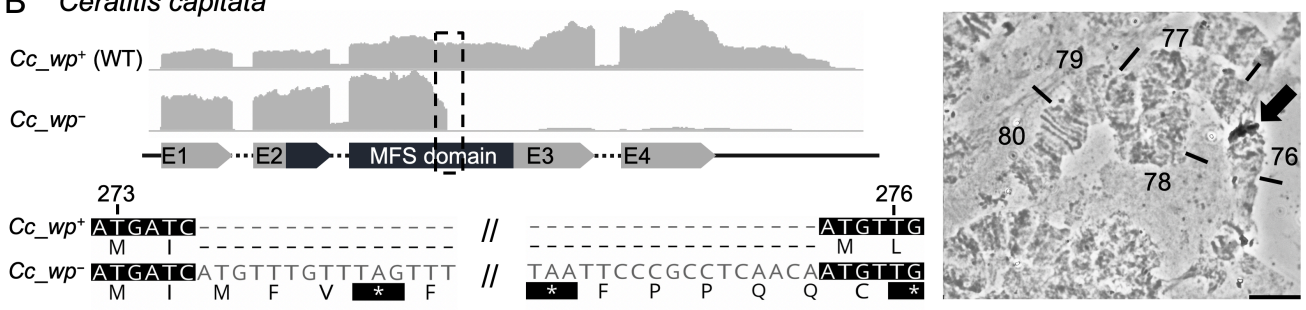

C Zeugodacus cucurbitae
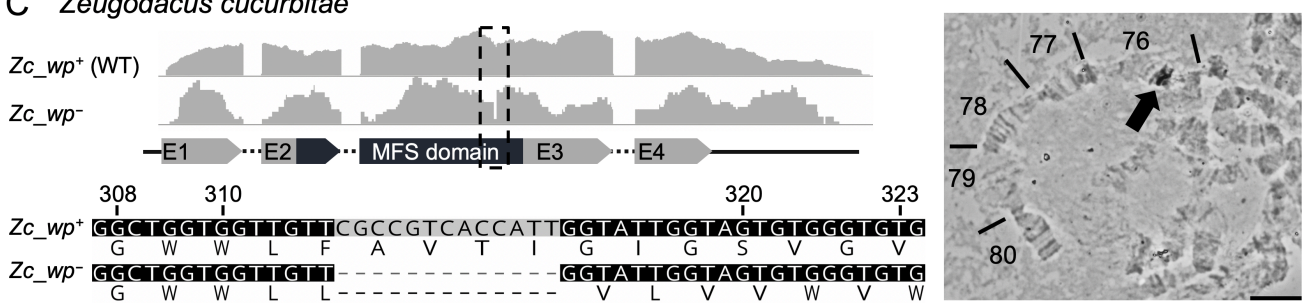

Fig. 3. Identification of the wp mutation in the transcriptomes of $B$. dorsalis, $C$. capitata and $Z$.

cucurbitae. The grey graphs show expression profiles from the candidate wp loci in WT $\left(w p^{+}\right)$and mutant ( $\left.w p^{-}\right)$flies at the immobile pupae stages of (A) B. dorsalis, (B) C. capitata and (C) Z. cucurbitae. The gene structure (not drawn to scale) is indicated below as exons (arrows labelled E1-E4) and introns (dashed lines). The positions of independent $w p$ mutations (Bd: 37 bp deletion, $C c$ : approximate 8,150 bp insertion, $Z c$ : 13 bp deletion) are marked with black boxes in the expression profiles and are shown in detail below the gene models based on de novo assembly of RNAseq data from WT and white pupae phenotype individuals. In situ hybridization on polytene chromosomes (right) confirmed the presence of the wp locus on the right arm of chromosome 5 in all three species (arrows in micrographs).

CRISPR/Cas9 knockout of a Major Facilitator Superfamily gene in B. tryoni and C. capitata causes the white pupae phenotype.

An analogous $B$. dorsalis wp- mutation was developed in $B$. tryoni by functional knockouts of the putative Bt_wp using the CRISPR/Cas9 system. A total of 591 embryos from the Ourimbah laboratory strain were injected using two guides with recognition sites in the first coding exon of this gene (Fig. 4A). Injected embryos surviving to adulthood $(n=19,3.2 \%)$ developed with either wild type brown $(n=12)$ or somatically mosaic white-brown puparia $(n=7$, Supplementary Fig. 5). Surviving $\mathrm{G}_{0}$ adults were individually backcrossed into the Ourimbah strain, resulting in potentially $w p^{+1-(C R I S P R)}$ heterozygous brown pupae (Fig. 4C). Five independent $G_{0}$ crosses were fertile (three mosaic white-brown and two brown pupae phenotypes), $G_{1}$ offspring were sibling mated and visual inspection of $G_{2}$ progeny revealed that three families contained white pupae individuals. Four distinct frameshift mutations were observed in screened $\mathrm{G}_{2}$ progeny (Fig. $4 \mathrm{~A}$ ) suggesting functional $\mathrm{KO}$ of putative Bt_wp is 
sufficient to produce the white pupae phenotype in $B$. tryoni. Capillary sequencing of cloned Bt_MFS amplicons revealed deletions ranging from a total of $4 \mathrm{bp}$ to $155 \mathrm{bp}$, summed across the two guide recognition sites, introducing premature stop codons.

In C. capitata, CRISPR/Cas9 gene editing was used to knockout the orthologous gene and putative Cc_wp, LOC101451947, to confirm that it causes a wp- phenotype. A mix of recombinant Cas9 protein and the gRNA_MFS, targeting the third exon and thereby the MFS domain of the presumed Cc_wp CDS (Fig. 4B), was injected into 588 Egll WT embryos of which 96 developed to larvae and 67 pupated. All injected $G_{0}$ pupae showed brown pupal color. In total, $29 \mathrm{G}_{0}$ males and 34 females survived to adulthood (9.3\%) and were backcrossed individually or in groups (see material and methods) to a strain carrying the naturally occurring white pupae mutation (wp-(nat); strain \#1402_22m1B $)^{24}$ (Fig. 4D). As white pupae is known to be monogenic and recessive in $C$. capitata, this complementation assay was used to reveal whether the targeted gene is responsible for the naturally occurring white pupae phenotype or if the mutation is located in a different gene. $G_{1}$ offspring would only show white pupae phenotypes if $C c \_w p$ was indeed the white pupae gene, knocked-out by the CRISPR approach, and complemented by the natural mutation through the backcross (wp(nat)-(CRISPR) $)$. In the case that the $C c_{-} w p$ is not the gene carrying the natural $w p^{-}$mutation, a brown phenotype would be observed for all offspring. Here, five out of 13 crosses, namely M1, M3, F2, F3, and F4, produced white pupae phenotype offspring. The crosses generated 221, $159,70,40$, and $52 \mathrm{G}_{1}$ pupae, of which $10,30,16,1$, and 1 pupa respectively, were white. Fifty-seven flies emerged from white pupae were analyzed via non-lethal genotyping, and all of them showed mutation events within the target region. Overall, eight different mutation events were seen, including deletions ranging from 1-9 bp and a 46 bp deletion combined with a 2 bp insertion (Fig. 4B). Five mutation events (B, D, E, G, H) caused frameshifts and premature stop codons. The remaining three $(A, C, F)$, however, produced deletions of only one to three amino acids. To make $w p^{\text {-(CRISPR) }}$ mutations homozygous, mutants were either inbred (mutation C) (Fig. 4D) or outcrossed to WT Egll (mutation A-H), both in groups according to their genotype. This demonstrated that $C c \_w p$ is the gene carrying the $w p^{- \text {(nat), }}$ and that even the loss of a single amino acid without a frameshift at this position can cause the white pupae phenotype. Offspring from outcrosses of mutation $A, D$, and $H$, as well as offspring of the inbreeding (mutation $C$ ), were genotyped via PCR, and $w p^{+1-(C R I S P R)}$ and $w p^{-}$ (CRISPR)-(CRISPR) positive flies were inbred to establish pure new white pupae lines. 

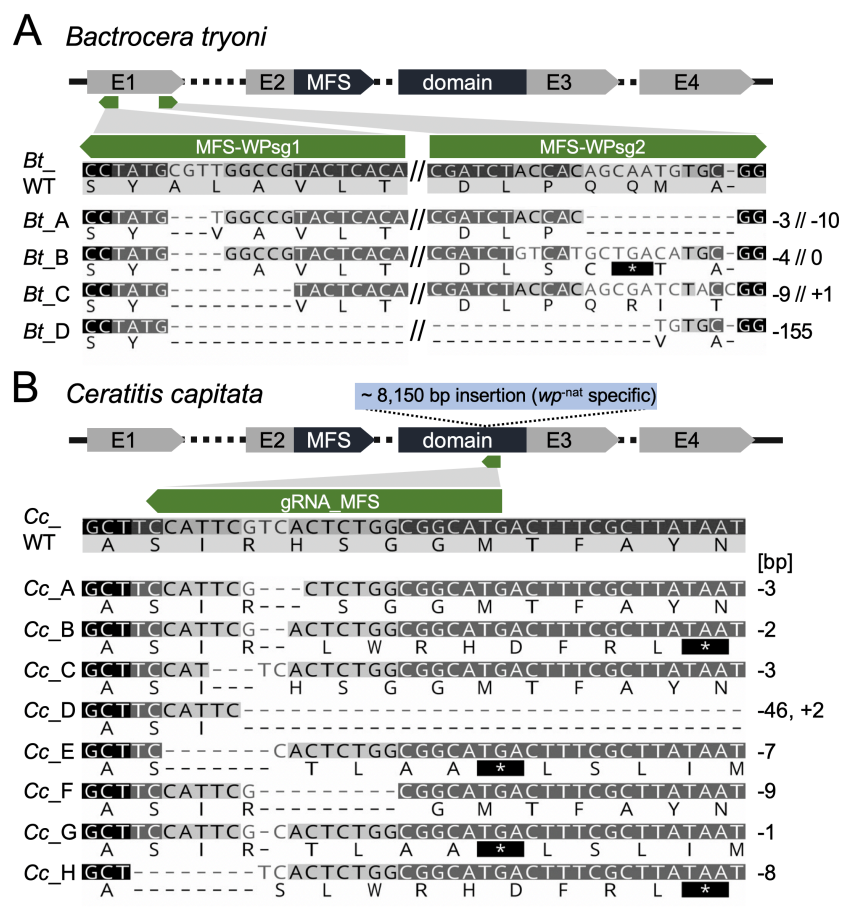

C Bactrocera tryoni
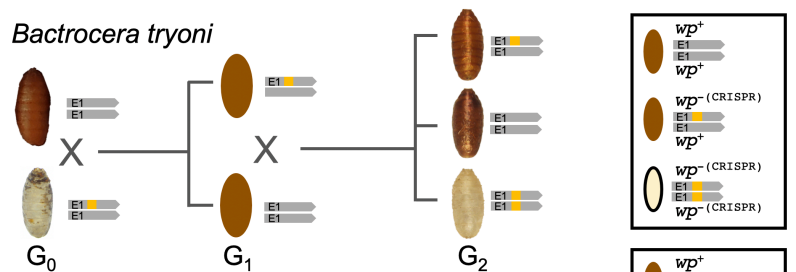

D Ceratitis capitata

Fig. 4. CRISPR/Cas9-based generation of homozygous wp-(CRISPR) lines in $B$. tryoni and $C$. capitata. A schematic structure of the wp CDS exons (E1, E2, E3, E4) including the MFS domain in $B$. tryoni $(\mathrm{A})$ and $C$. capitata $(\mathrm{B})$ are shown. Positions of gRNAs targeting the first and third exon in $B$. tryoni and $C$. capitata, respectively, are indicated by green arrows. Nucleotide and amino acid sequences of mutant wp alleles identified in $\mathrm{G}_{1}$ individuals are compared to the WT reference sequence in B. tryoni (A) and C. capitata (B). Deletions are shown as dashes, alterations on protein level leading to premature stop codons are depicted as asterisks highlighted in black. Numbers on the right side

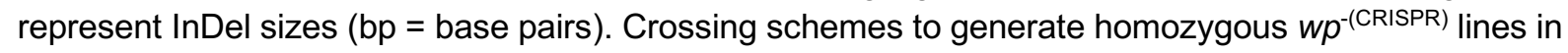
$B$. tryoni (C) and C. capitata (D) show different strategies to generate wp strains. Bright field images of empty puparia are depicted for both species. Genotype schematics and corresponding PCR analysis (for C. capitata) validating the presence CRISPR-induced (orange) and natural (blue, for $C$. capitata) wp mutations are shown next to the images of the puparia. (C) Injected $\mathrm{G}_{0} B$. tryoni were backcrossed to the Ourimbah laboratory strain resulting in uniformly brown $\mathrm{G}_{1}$ offspring (depicted as illustration because no images were acquired during $G_{1}$ ). $G_{1}$ inbreeding led to $G_{2}$ individuals homozygous for the white pupae phenotype. (D) Injected WT $\mathrm{G}_{0}$ flies were crossed to flies homozygous for the naturally occurring $w p^{-}$allele ( $w p^{- \text {(nat) }) . ~} w p^{- \text {(nat) }}$ (457 bp amplicon) and $w p^{- \text {(CRISPR) }}$ or WT (724 bp amplicon) alleles were identified by multiplex PCR (left lane; $L=$ NEB 2log ladder). White pupae phenotypes in $G_{1}$ indicated positive CRISPR events. $G_{2}$ flies with a white pupae phenotype that were homozygous for $w p^{-(C R I S P R)}$ allele were used to establish lines. 


\section{Discussion}

White pupae (wp) was first identified in C. capitata as a spontaneous mutation and was subsequently adopted as a phenotypic marker of fundamental importance for the construction of GSS for $S T^{6,9}$. Full penetrance expressivity and recessive inheritance rendered $w p$ the marker of choice for GSS construction in two additional tephritid species, $B$. dorsalis and $Z$. cucurbitae ${ }^{11,12}$, allowing automated sex sorting based on pupal color. This was only possible because spontaneous wp mutations occur at relatively high rates either in the field or in mass rearing facilities and can easily be detected ${ }^{6,9}$. Despite the easy detection and establishment of wp mutants in these three species, similar mutations have not been detected in other closely or distantly related species such as B. tryoni, B. oleae, or Anastrepha ludens, despite large screens being conducted. In addition to being a visible GSS marker used to separate males and females, the wp phenotype is also important for detecting and removing recombinants in cases where sex separation is based on a conditional lethal gene such as the $t s /$ gene in the medfly VIENNA 7 or VIENNA 8 GSS $^{6,7}$. However, it took more than 20 years from the discovery and establishment of the wp mutants to the large-scale operational use of the medfly VIENNA 8 GSS for SIT applications ${ }^{6,9}$ and the genetic nature of the wp mutation remained unknown. The discovery of the underlying wp mutations and the availability of CRISPR/Cas genome editing would allow the fast recreation of such phenotypes and sexing strains in other insect pests. Isolation of the wp gene would also facilitate future efforts towards the identification of the closely linked $t s /$ gene.

Using an integrated approach consisting of genetics, cytogenetics, genomics, transcriptomics and bioinformatics, we identified the white pupae $(w p)$ genetic locus in three major tephritid agricultural pest species, B. dorsalis, C. capitata, and Z. cucurbitae.

Our study clearly shows the power of employing different strategies for gene discovery, one of which was species hybridization. In Drosophila, hybridization of different species has played a catalytic role in the deep understanding of species boundaries and the speciation processes, including the evolution of mating behavior and gene regulation ${ }^{25-29}$. In our study, we took advantage of two closely related species, $B$. dorsalis and $B$. tryoni, which can produce fertile hybrids and be backcrossed for consecutive generations. This allowed the introgression of the wp mutant locus of $B$. dorsalis into $B$. tryoni, resulting in the identification of the introgressed region, including the causal wp mutation via whole-genome resequencing and advanced bioinformatic analysis.

In C. capitata, we exploited two essential pieces of evidence originating from previous genetic and cytogenetic studies: the localization of $w p$ to region $59 \mathrm{~B}$ and $76 \mathrm{~B}$ on chromosome its position close to the right breakpoint of the large inversion D53 ${ }^{6}$. This data prompted us to undertake a comparative genomic approach to identify the exact position of the right 
breakpoint of the D53 inversion, which would bring us in the vicinity of the wp gene. Coupled with comparative transcriptomic analysis, this strategy ensured that the analysis indeed tracked the specific wp locus on the right arm of chromosome 5, instead of any mutation in another, random locus which may participate in the pigmentation pathway and therefore result in the same phenotype. Functional characterization via CRISPR/Cas9-mediated knockout resulted in the establishment of new white pupae strains in C. capitata and B. tryoni and confirmed that this gene is responsible for the pupal coloration in these tephritid species. Interestingly, the wp phenotype is based on three independent and very different natural mutations of this gene, a rather large and transposon-like insertion in C. capitata, but only small deletions in the two other tephritids, B. dorsalis and Z. cucurbitae. In medfly, however, CRISPR-induced in-frame deletions of one or three amino acids in the MFS domain were sufficient to induce the wp phenotype, underlining the importance of this domain for correct pupal coloration.

It is worth noting, that in the first stages of this study, we employed two additional approaches, which did not allow us to successfully narrow down the wp genomic region to the desired level. The first was based on Illumina sequencing of libraries produced from laser micro-dissected $(Y ; 5)$ mitotic chromosomes that carry the wild type allele of the wp gene through a translocation from the fifth chromosome to the $Y$. This dataset from the medfly VIENNA 7 GSS was comparatively analyzed to wild type (Egypt II) $Y$ and $X$ chromosomes, and the complete genomes of Egypt II, VIENNA $7^{\text {D53- }}$ GSS, and a D53 inversion line in an attempt to identify the chromosomal breakpoints of the translocation and/or inversion, which are close to the wp locus (Supplementary Table 2). However, this effort was not successful due to the short Illumina reads and the lack of a high-quality reference genome. The second approach was based on individual scale whole-genome resequencing/genotyping, and identifying fixed loci associated with pupal color phenotypes, which complemented the QTL analysis $^{19}$. Seven loci associated with SNPs and larger deletions linked to the white pupae phenotype were analyzed based on their respective mutations and literature searches for their potential involvement in pigmentation pathways. However, we could not identify a clear link to the pupal coloration as shown by in silico, molecular, and in situ hybridization analysis (Supplementary Fig. 6 and 7, Supplementary Table 3).

375 The wp gene is a member of a major facilitator superfamily (MFS). Orthologs of white pupae 376 are present in 146 of 148 insect species aggregated in OrthoDB v $9^{20}$ present in all orders and 377 single copy in 133 species. Furthermore, $w p$ is included in the benchmarking universal single copy ortholog (BUSCO) gene set for insecta and according to OrthoDB $v 10^{31}$ has a below average evolutionary rate (0.87, OrthoDB group 42284at50557) suggesting an important and evolutionarily conserved function (Supplementary Fig. 8). Its ortholog in Bombyx mori, mucK, 
peak expression is during the prepupal stage after the larva has committed to pupation ${ }^{33}$,

383 which is the stage where pupal cuticle sclerotization and melanization occurs. It is known that

384 the insect cuticle consists of chitin, proteins, lipids and catecholamines, which act as cross-

385 linking agents thus contributing to polymerization and the formation of the integument ${ }^{34}$.

386 Interestingly, the sclerotization and melanization pathways are connected and this explains

387 the different mechanical properties observed in different medfly pupal color strains with the

388 "dark" color cuticles to be harder than the "brown" ones and the latter harder than the "white"

389 color ones ${ }^{35}$. The fact that the white pupae mutants are unable to transfer catecholamines

390 from the haemolymph to the cuticle is perhaps an explanation for the lack of the brown 391 pigmentation ${ }^{13}$.

392 The discovery of the long-sought wp gene in this study and the recent discovery of the 393 Maleness-on-the-Y (MoY) gene, which determines the male sex in several tephritids ${ }^{36}$, opens 394 the way for the development of a 'generic approach' for the construction of GSS for other 395 species. Using CRISPR/Cas-based genome editing approaches, we can: (a) induce mutations 396 in the wp orthologues of SIT target species and establish lines with wp phenotype and (b) link 397 the rescue alleles as closely as possible to the MoY region. Given that the wp gene is present 398 in diverse insect species including agricultural insect pests and mosquito disease vectors, this 399 approach would allow more rapid development of GSS in SIT target species. In principle, these 400 GSS will have higher fertility compared to the semi-sterile translocation lines ${ }^{6}$. In addition, 401 these new generation GSS will be more stable since the rescue allele will be tightly linked to 402 the male determining region thus eliminating recombination which can jeopardize the genetic 403 integrity of any GSS. The concept of the 'generic approach' can also be applied in species 404 which lack a typical Y chromosome such as Aedes aegypti and Aedes albopictus. In these 405 species, the rescue allele should be transferred close to the male determining gene (Nix) and 406 the $\mathrm{M} \mathrm{locus}^{37,38}$. It is hence important for this 'generic approach' to identify regions close 407 enough to the male determining loci to ensure the genetic stability of the GSS and to allow the 408 proper expression of the rescue alleles. In the present study, we have already shown that 409 CRISPR/Cas9-induced mutations resulting in the white pupae phenotype can be developed 410 in SIT target species and the resulting strains provide already new opportunities for GSS 411 based on visible markers. 
414 All sequence libraries prepared during this study are publicly available on NCBI within the ENA

415 BioProject PRJEB36344/ERP119522 (accession numbers ERS4426857 - ERS4426873, 416 ERS4426994 - ERS4427029, ERS4519515, ERS4547590 - ERS4547593; see

417 Supplementary Table 1) and the BioProject PRJNA629430 (SRA accessions SRR11649127 418 - SRR11649132; see Supplementary Fig. 6).

419

420 Insect rearing. C. capitata, B. dorsalis and Z. cucurbitae fly strains were maintained at $42125 \pm 1^{\circ} \mathrm{C}, 48 \% \mathrm{RH}$ and $14 / 10 \mathrm{~h}$ light/dark cycle. They were fed with a mixture of sugar and yeast 422 extract (3v:1v) and water. Larvae were reared on a gel diet, containing carrot powder (120 g/l), 423 agar $(3 \mathrm{~g} / \mathrm{l})$, yeast extract $(42 \mathrm{~g} / \mathrm{l})$, benzoic acid $(4 \mathrm{~g} / \mathrm{l}), \mathrm{HCl}(25 \%, 5.75 \mathrm{ml} / \mathrm{l})$ and ethyl-4424 hydroxybenzoate $(2.86 \mathrm{~g} / \mathrm{l})$. Flies were anesthetized with $\mathrm{N}_{2}$ or $\mathrm{CO}_{2}$ for screening, sexing, and 425 the setup of crosses. To slow down the development during the non-lethal genotyping process (C. capitata), adult flies were kept at $19^{\circ} \mathrm{C}, 60 \% \mathrm{RH}$, and $24 \mathrm{~h}$ light for this period (1-4 d).

B. tryoni flies were obtained from New South Wales Department of Primary Industries (NSW DPI), Ourimbah, Australia and reared at $25 \pm 2^{\circ} \mathrm{C}, 65 \pm 10 \% \mathrm{RH}$ and $14 / 10 \mathrm{~h}$ light/dark cycle. Flies were fed with sugar, Brewer's yeast and water and larvae were reared on a gel diet, containing Brewer's yeast (204 g/l), sugar (121 g/l), methyl p-hydroxy benzoate $(2 \mathrm{~g} / \mathrm{l})$, citric acid $(23 \mathrm{~g} / \mathrm{l})$, wheat germ oil $(2 \mathrm{~g} / \mathrm{l})$, sodium benzoate $(2 \mathrm{~g} / \mathrm{l})$ and agar $(10 \mathrm{~g} / \mathrm{l})$.

Introgression and bioinformatic identification of a natural wp mutation from $B$. dorsalis

in B. tryoni. Interspecific crosses between B. tryoni and white pupae B. dorsalis were carried out between male $B$. tryoni $\left(w p^{+\mid+}\right)$and female $B$. dorsalis $\left(w p^{-1}\right)$. The $G_{1} w p^{+-}$hybrids developed with brown puparia and were mass crossed. $\mathrm{G}_{2}$ wp $p^{-t}$ females were backcrossed into $B$. tryoni $w p^{+++}$males and backcrossing was then repeated for five additional times to produce the white pupae Bactrocera Introgressed Line (BIL, Supplementary Fig. 1).

Genome sequencing using Illumina NovaSeq (2 x 150 bp, Deakin University) was performed on a single male and female from the $B$. dorsalis wp strain, $B$. tryoni, and the $B I L$ $(\sim 26 X)$ and two pools of five BIL individuals ( 32X). Quality control of each sequenced library

carried

out

using

FastQC

v0.11.6

443 (https://www.bioinformatics.babraham.ac.uk/projects/fastqc/) and aggregated using ngsReports $^{39}$ v1.3. Adapter trimming was carried out using Trimmomatic v0.38 (https://academic.oup.com/bioinformatics/article/30/15/2114/2390096) and paired reads were mapped to the $B$. dorsalis reference genome (GCF_000789215.1) using NextGenMap ${ }^{40}$ v0.5.5 under default settings. Mapped data were sorted and indexed using SAMtools, and 
449 (https://github.com/broadinstitute/picard). Genotypes were called on single and pooled 450 libraries separately with ploidy set to two and ten respectively using Freebayes ${ }^{41}$ v1.0.2. Each 451 strain was set as a different population in Freebayes. Genotypes with less than five genotype 452 depth were set to missing and sites with greater than $20 \%$ missing genotypes or indels filtered 453 out using BCFtools ${ }^{42}$ v1.9. Conversion to the genomic data structure (GDS) format was carried 454 out using SeqArray ${ }^{43}$ v1.26.2 and imported into the $R$ package geaR v0.1 455 (https://github.com/CMWbio/geaR) for population genetic analysis.

Single copy orthologs were identified in the $B$. dorsalis reference annotated proteins (NCBI Bactrocera dorsalis Annotation Release 100) with BUSCO ${ }^{44,45}$ v3 using the dipteran gene set $^{44}$. Nucleotide alignments of each complete single copy ortholog were extracted from the called genotype set using geaR v0.1 and gene trees built using RAxML ${ }^{46}$ v8.2.10 with a GTR+G model. Gene trees were then imported into Astral III v5.1.1 ${ }^{47}$ for species tree estimation. Genome scans of absolute genetic divergence $\left(d_{X Y}\right)$, nucleotide diversity $(\pi)$, and the $\mathrm{f}$ estimator $\widehat{f_{d}}$ were carried out using geaR v0.1. Two levels of analysis were carried out: i) genome wide scans of non-overlapping $100 \mathrm{~kb}$ windows and ii) locus scans of $10 \mathrm{~kb}$ tiled windows. Local phylogenies were built for nucleotide alignments of non-overlapping $1 \mathrm{~kb}$ windows using RAxML v8.2.10 with a GTR+G model and topology weighting was calculated using TWISST ${ }^{48}$.

Introgressed regions (i.e. candidate wp loci) were identified by extracting windows in the genome wide scan with topology weighting and fd greater than 0.75 and visually inspecting the 'locus scan' data set for $\mathrm{d}_{\mathrm{XY}}$, $\mathrm{fd}$ and topology weighting patterns indicative of introgression. Nucleotide alignments of all genes within candidate $B$. dorsalis introgressed regions were extracted from the GDS using geaR and visually inspected for fixed mutations in $B$. dorsalis $w p$, BIL individuals and the two BIL pools. Candidate genes were then searched by tBLASTn against the $D$. melanogaster annotated protein set to identify putative functions and functional domains were annotated using $\mathrm{HMMer}^{49}$. Mapped read depth was calculated around candidate regions using SAMtools ${ }^{50}$ depth v1.9 and each sample's read depth was normalized to the sample maximum to inspect putative deletions. Called genotypes were confirmed by de novo genome assembly of the $B$. dorsalis wp genome using MaSuRCA ${ }^{51}$ v3.3 under default settings. The de novo scaffold containing LOC105232189 was identified using the BLASTn algorithm. In silico exon-intron boundaries were then manually annotated in Geneious ${ }^{52}$ v11. locus in C. capitata.

484 Egypt II (Egll) is a wild type laboratory strain. D53 is a homozygous strain with an irradiation- 
(50B-59C on the trichogen cells polytene chromosome map). VIENNA 7 and VIENNA 8 are two GSS in which two $(Y ; 5)$ translocations, on the region 58B and 52B of the trichogen cells polytene chromosome map respectively, have resulted to the linkage of the wild type allele of the $w p$ and $t s /$ genes to the male determining region of the $Y$ chromosome. Thus, VIENNA 7 and VIENNA 8 males are heterozygous in the wp and $t s /$ loci but phenotypically wild type while VIENNA 7 and VIENNA 8 females are homozygous for the mutant alleles and phenotypically white pupae and they die when exposed at elevated temperatures. The VIENNA 7 and VIENNA 8 GSS can be constructed with and without the D53 inversion (VIENNA $7 / 8^{\text {D53+ }}$ or ${ }^{\text {D53- }}$ ). When the GSS have the inversion, females are homozygous $\left({ }^{\mathrm{D} 53+\left.\right|^{+}}\right)$for D53 while males are heterozygous $\left({ }^{\mathrm{D} 53+1}\right)^{6,8,16}$.

Whole genome sequencing of $\mathrm{C}$. capitata strains

High-molecular-weight (HMW) DNA was extracted from C. capitata lines (males and females of the wild type Egll strain, the VIENNA $7^{\text {D53-- }}$ and VIENNA $8^{\text {D53-- }}$ GSS and the inversion line D53) and sequenced. Freshly emerged, virgin and unfed males and females were collected from all strains. For 10X Genomics linked read and Nanopore sequencing, the HMW was prepared as follows: twenty individuals of each sex and strain were pooled, ground in liquid nitrogen, and HMW DNA was extracted using the QIAGEN Genomic tip 100/G kit (Qiagen, Germany). For PacBio Sequel an Egll line was created with single pair crossing and subsequent sibling-mating for six generations. In all generations adult and larval diet contained $100 \mu \mathrm{g} / \mathrm{ml}$ tetracycline. HMW DNA from $\mathrm{G}_{6}$ individuals was prepared as follows: five males from this Egll line were pooled and ground in liquid nitrogen, and HMW DNA was extracted using the phenol/chloroform Phase Lock Gel ${ }^{\mathrm{TM}}$ tubes (QuantaBio) ${ }^{53}$. DNA for Illumina applications was extracted from individual flies (Supplementary Table 1).

PacBio de novo sequencing: samples were purified with AMPure beads (Beckman Coulter,

511 UK) (0.6 volumes) and QC checked for concentration, size, integrity and purity using Qubit 512 (Qiagen, UK), Fragment Analyser (Agilent Technologies) and Nanodrop (Thermo Fisher) machines. The samples were then processed without shearing using the PacBio Express kit

5141 for library construction and an input of $4 \mu \mathrm{g}$ DNA following the manufacturer's protocol. The 515 final library was size-selected using the Sage Blue Pippin (Sage Sciences) $0.75 \%$ cassette U1 516 marker in the range of $25-80 \mathrm{~kb}$. The final library size and concentrations were obtained on 517 the Fragment Analyser before being sequenced using the Sequel 12.1 chemistry with V4 518 primers at a loading on plate concentration of $6 \mathrm{pM}$ and $10 \mathrm{~h}$ movie times.

For Nanopore sequencing, the ligation sequencing kits SQK-LSK109 or SQK-RAD004 were used as recommended by the manufacturer (Oxford Nanopore Technologies, Oxford,

521 United Kingdom). Starting material for the ligation library preparation were 1 - $1.5 \mu \mathrm{g} \mathrm{HWW}$ 522 gDNA for the ligation libraries and $400 \mathrm{ng}$ for the rapid libraries. The prepared libraries were 
523 loaded onto FLO-PRO002 (R9.4) flow cells. Data collection was carried out using a

524 PromethION Beta with live high accuracy base calling for up to $72 \mathrm{~h}$ and with mux scan

525 intervals of $1.5 \mathrm{~h}$. Each sample was sequenced at least twice. Data generated were $7.7 \mathrm{~Gb}$

526 for Egll male, $31.09 \mathrm{~Gb}$ for D53 male, $26.72 \mathrm{~Gb}$ for VIENNA $7^{\text {D53-- }}$ male, and $24.83 \mathrm{~Gb}$ for

527 VIENNA $8^{\text {D53-- }}$ male. Run metrics are shown in Supplementary Table 5.

528 The PacBio data were assembled using $\mathrm{CANU}^{54}$ with two parameter settings: the first to 529 avoid haplotype collapsing (genomeSize $=500 \mathrm{~m}$ corOutCoverage=200 "batOptions=-dg $3-\mathrm{db}$

$5303-\mathrm{dr} 1$-ca $500-\mathrm{cp} \mathrm{50")} \mathrm{and} \mathrm{the} \mathrm{second} \mathrm{to} \mathrm{merge} \mathrm{haplotypes} \mathrm{together} \mathrm{(genomeSize}=500 \mathrm{~m}$ corOutCoverage $=200$ correctedErrorRate $=0.15)$. The genome completeness was assessed with BUSCO ${ }^{44,45}$ v3 using the dipteran gene $\operatorname{set}^{44}$. The two assemblies were found to be "duplicated" due to alternative haplotypes. To improve the contiguity and reduce duplication haploMerger2 was used ${ }^{55}$. The new assembly was then retested with BUSCO v3 and used scaffolding. Phase Genomics ${ }^{\mathrm{TM}} \mathrm{Hi}-\mathrm{C}$ libraries were made by Phase genomics from males $(n=2)$ of the same family used for PacBio sequencing. Initial scaffolding was completed by

537 Phase Genomics, but edited using the Salsa ${ }^{56}$ and 3D-DNA (https://github.com/theaidenlab/3d-dna) software. The resulting scaffolds were allocated a chromosome number using chromosome specific marker described previously ${ }^{16}$. Specific attention was made to the assembly and scaffolding of chromosome 5. Two contig misassemblies were detected by the $\mathrm{Hi}-\mathrm{C}$ data and fitted manually. The new assembly

542 (Egll_Ccap3.2.1) was then validated using the $\mathrm{Hi}-\mathrm{C}$ data. Genes were called using the

543 Funannotate software making use of the Illumina RNAseq data generated by this project; mRNA mapping to the genome is described below.

D53 breakpoint analysis in C. capitata

547 To identify possible breakpoint positions, the Nanopore D53 fly assembly contig_531 was 548 mapped onto the Egll_scaffold_5 (from the Egll_CCAP3.2_CANU_Hi-C_scaffolds.fasta 549 assembly) using MashMap v2.0 (https://github.com/marbl/MashMap). This helped to visualize 550 the local alignment boundaries (Supplementary Fig. 9). MashMap parameters were set to 551 kmer size $=16$; window size $=100$; segment length $=500$; alphabet $=$ DNA; percentage identity threshold $=95 \%$; filter mode $=$ one-to-one .

Subsequent to this, and to help confirm the exact location of the identified breakpoints, minimap2 (v2.17, https://github.com/lh3/minimap2) was used to align D53 as well as VIENNA $8^{\text {D53-- }}$ and VIENNA $7^{\text {D53-- }}$ Nanopore reads onto the Egll scaffold_5 reference (Supplementary Fig. 9). Minimap2 parameters for Nanopore reads were: minimap2 -x map-ont -A 1 -a --MD L -t 40. Samtools (v1.9, https://github.com/samtools/samtools) was used to convert the alignment.sam to .bam and prepare the alignment file to be viewed in the Integrative Genomics Viewer (IGV, http://software.broadinstitute.org/software/igv/). The expectation was to see a 
560 leftmost breakpoint in D53 read set alignments but not in VIENNA $8^{\text {D53-- }}$ and VIENNA $7^{\text {D53-- }}$ 561 when compared to the Egll reference (Supplementary Fig. 9). Due to an assembly gap in the

562 Egll scaffold_5 sequence, the exact location of the leftmost inversion breakpoint was not 563 conclusive using this approach.

564 A complementary approach was then used to facilitate detection of the leftmost inversion

565

566

567

568

569

570

571

572

573

574

575

576

577

578

579

580

581

582

583

584

585

586

587

588

589

590

591

592

593

594

595

596 breakpoint in the D53 inversion line. Minimap2 was again used, but here D53 contig_531 was used as reference for the mapping of Egll male PacBio reads as well as VIENNA ${ }^{8 D 53-\mid-}$ male and VIENNA 7 D53-|- male Nanopore reads (Supplementary Fig. 10). Minimap2 parameters for PacBio reads were: minimap2 -x map-pb -A 1 -a --MD -L -t 40. Minimap2 parameters for Nanopore reads were: minimap2 -x map-ont -A 1 -a --MD -L -t 40. Samtools (v1.9, https://github.com/samtools/samtools) was used to convert the alignment .sam to .bam and prepare the alignment file to be viewed in the Integrative Genomics Viewer (IGV, http://software.broadinstitute.org/software/igv/). The expectation was to see a common breakpoint for all three of the above read set alignments when compared to the D53 genome in the area of the inversion. Position 3,055,294 was identified in the D53 contig_531 as the most likely leftmost breakpoint.

To determine the rightmost breakpoint, D53 male and VIENNA $8^{\text {D53-l- }}$ and VIENNA $7^{\text {D53-1- }}$ male nanopore reads were aligned on the Egll_scaffold_5 sequence. The expectation was to

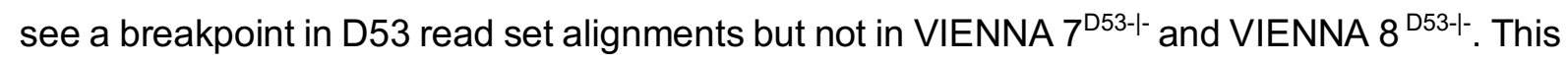
is the case here, since read alignments coming from both sides of the inversion are truncated at one position (Supplementary Fig. 9). Findings from genome version Egll_Ccap3.2 were extrapolated to the manually revised genome version Egll_Ccap3.2.1.

Predicted D53 inversion breakpoints were verified via PCRs in Egll, D53, and VIENNA $7^{\text {D53+ }}$ GSS male and female flies, using PhusionFlash Polymerase in a $10 \mu \mathrm{l}$ reaction volume $\left[98^{\circ} \mathrm{C}\right.$, $10 \mathrm{~s} ; 30$ cycles of $\left(98^{\circ} \mathrm{C}, 1 \mathrm{~s} ; 56^{\circ} \mathrm{C}, 5 \mathrm{~s} ; 72^{\circ} \mathrm{C}, 35 \mathrm{~s}\right) ; 72^{\circ} \mathrm{C}, 1 \mathrm{~min}$ ] (Supplementary Fig. 4). The primer pair for the right breakpoint was designed based on Egll sequence information, primers for the left breakpoint were designed based on D53 sequence information. The wild type status of chromosome 5 (Egll male and female, VIENNA $7^{\text {D53+|- }}$ male) was amplified using primer pairs P_1794 and P_1798 (1,950 bp) and P_1795 and P_1777 (690 bp) (Supplementary Table

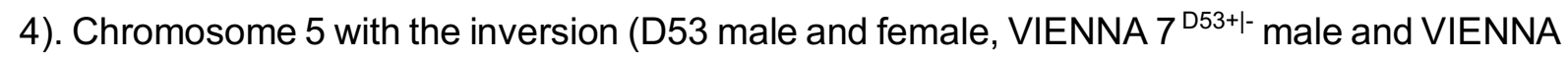
$7^{\text {D53+|+ }}$ female) was verified using primer pairs $P_{-} \_1777$ and $P \_1798(1,188 \mathrm{bp})$ and $P_{-} 1794$ and P_1795 (1,152 bp) and amplicon sequencing (Macrogen Europe, Amsterdam).

RNA extraction for transcriptomic analysis of C. capitata, B. dorsalis and Z. cucurbitae Samples of C. capitata, B. dorsalis and Z. cucurbitae species were collected for RNA extraction (Supplementary Table 1) at 3rd instar larval and pre-pupal stages. Total RNA was extracted by homogenizing three larvae of $C$. capitata and $B$. dorsalis and a single larvae of 
597 Z. cucurbitae in liquid nitrogen, and then using the RNeasy Mini kit (Qiagen). Three replicates

598 per strain and time point were performed. mRNA was isolated using the NEBNext polyA 599 selection and the Ultra II directional RNA library preparation protocols from NEB and 600 sequenced on the Illumina NovaSeq 6000 using duel indexes as 150 bp paired end reads 601 (library insert $500 \mathrm{bp}$ ). Individual libraries were sequenced to provide $>1$ million paired end 602 reads per sample. Each replicate was then assembled separately using Trinity ${ }^{57}$. The 603 assembled transcripts from Trinity were mapped to the Ccap3.2 genome using minimap ${ }^{58}$ (parameters -ax splice:hq -uf). The Illumina reads were mapped with STAR ${ }^{59}$. IGV ${ }^{60}$ v 2.6 was used to view all data at a genomic and gene level. Given that the white pupae GSS $^{12,61}$ was used to collect samples for RNA extraction from single larvae of $Z$. cucurbitae, larval sex was confirmed by a maleness-specific PCR on the MoY gene of Z. cucurbitae ${ }^{36}$ using cDNA synthesized with the OneStep RT-PCR Kit (Qiagen) and the primer pair ZcMoY1F and ZcMoY1R (Supplementary Table 4) amplifying a 214 bp fragment. Conditions for a $25 \mu \mathrm{IPCR}$ reaction using the $1 \times$ Taq PCR Master Mix kit (Qiagen) were: $\left[95^{\circ} \mathrm{C}, 5 \mathrm{~min} ; 30\right.$ cycles of $\left(95^{\circ} \mathrm{C}\right.$, $\left.1 \mathrm{~min} ; 51^{\circ} \mathrm{C}, 1 \mathrm{~min} ; 72^{\circ} \mathrm{C}, 1 \mathrm{~min}\right) ; 72^{\circ} \mathrm{C}, 10 \mathrm{~min}$. Presence of a PCR product indicated a male sample. Each, male and female sample was a pool of three individuals. Three replicates per strain and time point were collected.

Functional and cytogenetic verification of medfly D53 inversion and tephritid wp genes. Polytene chromosomes for in situ hybridization were prepared from third-instar larvae salivary glands as described previously ${ }^{62}$. In brief, the glands were dissected in $45 \%$ acetic acid and placed on a coverslip in a drop of 3:2:1 solution (3 parts glacial acetic acid: 2 parts water: 1 part lactic acid) until been transparent (approximately $5 \mathrm{~min}$ ). The coverslip was picked up with a clean slide. After squashing, the quality of the preparation was checked by phase contrast microscope. Satisfactory preparations were left to flatten overnight at $-20^{\circ} \mathrm{C}$ and dipped into liquid nitrogen until the babbling stopped. The coverslip was immediately removed with razor blade and the slides were dehydrated in absolute ethanol, air dried and kept at room temperature.

Probes were prepared by PCR. Single adult flies were used to extract DNA with the Extract me kit (Blirt SA), following the manufacturer's protocol. NanoDrop spectrometer was used to assess the quantity and quality of the extracted DNA which was then stored at $-20^{\circ} \mathrm{C}$ until used. Primers (P1790/1791, P1821/1822, Pgd_probe_F/R, vg1_probe_F/R, Sxl_probe_F/R, y_probe_F/R, zw_probe_F/R, P1633/1634, Zc_F/R, Bd_F/R, P1395/1396, P1415/1416;

630 Supplementary Table 4) were designed for each targeted gene using Primer 3 and/or 631 Geneious Prime programs. PCR was performed in a $25 \mu$ l reaction volume using $12.5 \mu \mathrm{I}$ PCR 632 Master mix 2x Kit (Thermo Fisher Scientific), 60-80 ng DNA, and the following PCR settings $633\left[94^{\circ} \mathrm{C}, 5 \mathrm{~min} ; 35\right.$ cycles of $\left.\left(94^{\circ} \mathrm{C}, 45 \mathrm{~s} ; 56^{\circ} \mathrm{C}, 30 \mathrm{~s} ; 72^{\circ} \mathrm{C}, 90 \mathrm{~s}\right) ; 72^{\circ} \mathrm{C}, 1 \mathrm{~min}\right]$. 
The labelling of the probes was carried out according to the instruction manual of the Dig DNA labelling kit (Roche). In situ hybridization was performed as described previously ${ }^{63}$. In brief, before hybridization, stored chromosome preparations were hydrated by placing them for $2 \mathrm{~min}$ at each of the following ethanol solutions: $70 \%, 50 \%$ and $30 \%$. Then they were placed in $2 \times$ SSC at room temperature for $2 \mathrm{~min}$. The stabilization of the chromosomes was done by placing them in $2 \times \mathrm{SSC}$ at $65^{\circ} \mathrm{C}$ for 30 min, denaturing in $0.07 \mathrm{M} \mathrm{NaOH} 2 \mathrm{~min}$, washing in $2 \times$ SSC for $30 \mathrm{sec}$, dehydrating (2 $\mathrm{min}$ in $30 \%, 50 \%, 70 \%$ and $95 \%$ ethanol), and air drying. Hybridization was performed on the same day by adding $15 \mu \mathrm{l}$ of denatured probe (boiled for $10 \mathrm{~min}$ and ice-chilled). Slides were covered with a siliconized coverslip, sealed with rubber cement, and incubated at $45^{\circ} \mathrm{C}$ overnight in a humid box. At the end of incubation, the coverslip was floated off in $2 \times$ SSC and the slide washed in $2 \times S S C$ for $3 \times 20$ min at $53^{\circ} \mathrm{C}$.

After 5 min wash in Buffer $1(100 \mathrm{mM}$ tris- $\mathrm{HCl} \mathrm{pH} \mathrm{7.5/1.5} \mathrm{M} \mathrm{NaCl})$, the preparations were in Blocking solution (Blocking reagent $0.5 \%$ in Buffer 1) for $30 \mathrm{~min}$, and then washed for $1 \mathrm{~min}$ in Buffer 1. The antibody mix was added to each slide and a coverslip was added. Then the slides were incubated in a humid box for $45 \mathrm{~min}$ at room temperature, following $2 \times 15 \mathrm{~min}$ washes in Buffer 1, and a 2 min wash in detection buffer (100 mM Tris-HCl pH 9.5/ $100 \mathrm{mM}$ $\mathrm{NaCl}$ ). The color was developed with $1 \mathrm{ml}$ of NBT/BCIP solution during a 40 min incubation in the dark at room temperature. The removal of the NBT/BCIP solution was done by rinsing in water twice. Hybridization sites were identified using $40 x$ or $100 x$ oil objectives (phase or bright field) and a Leica DM 2000 LED microscope, with reference to the salivary gland chromosome maps $^{64}$. Well-spread nuclei or isolated chromosomes were photographed using a digital camera (Leica DMC 5400) and the LAS X software. All in situ hybridizations were performed at least in duplicates and at least 10 nuclei were analyzed per sample.

Gene editing and generation of homozygous wp strains in B. tryoni and C. capitata. For CRISPR/Cas9 gene editing in B. tryoni, purified Cas9 protein (Alt-R S.p. Cas9 Nuclease V3, \#1081058, $10 \mathrm{\mu g} / \mu \mathrm{l}$ ) and guide RNAs (customized Alt-R ${ }^{\circledR}$ CRISPR/Cas9 crRNA, 2 nmol and Alt-R CRISPR/Cas9 tracrRNA, \#1072532, 5nmol) were obtained from Integrated DNA Technologies (IDT) and stored at $-20^{\circ} \mathrm{C}$. The guide RNAs were individually resuspended to a $100 \mu \mathrm{M}$ stock solution with nuclease-free duplex buffer and stored at $-20^{\circ} \mathrm{C}$ before use. The two customized 20 bp crRNA sequences (Bt_MFS-1 and Bt_MFS-2) (Supplementary Table 4) were designed using CRISPOR ${ }^{65}$. Injection mixes for microinjection of $B$. tryoni embryos comprise of $300 \mathrm{ng} / \mu \mathrm{l}$ Cas 9 protein, $59 \mathrm{ng} / \mu \mathrm{l}$ of each individual crRNA, $222 \mathrm{ng} / \mu \mathrm{l}$ tracrRNA and $1 \mathrm{x}$ injection buffer $(0.1 \mathrm{mM}$ sodium phosphate buffer $\mathrm{pH} 6.8,5 \mathrm{mM} \mathrm{KCl})$ in a final volume of $10 \mu \mathrm{l}$. The guide RNA complex containing the two crRNAs and tracrRNA was initially prepared by heating the mix at $95^{\circ} \mathrm{C}$ for 5 min before cooling to room temperature. The Cas 9 enzyme along with the rest of the injection mix components were then added to the guide RNA 
671 complex and incubated at room temperature for 5 minutes to assemble the RNP complexes.

672 Microinjections were performed in B. tryoni Ourimbah laboratory strain embryos that were

673 collected over 1 hour time period and prepared for injection as previously described ${ }^{22}$.

674 Injections were performed under paraffin oil using borosilicate capillary needles (\#30-0038,

675 Harvard Apparatus) drawn out on a Sutter P-87 flaming/brown micropipette puller and

676 connected to an air-filled $20 \mathrm{ml}$ syringe, a manual MM-3 micromanipulator (Narishige) and a

677 CKX31-inverted microscope (Olympus). Microscope slides with the injected embryos were

678 placed on agar in a Petri dish that was then placed in a vented container containing moist

679 paper towels at $25^{\circ} \mathrm{C}\left( \pm 2^{\circ} \mathrm{C}\right)$. Hatched first instar larvae were removed from the oil and

680 transferred to larval food. Individual $\mathrm{G}_{0}$ flies were crossed to six virgin flies from the Ourimbah

681 laboratory strain and eggs were collected overnight for two consecutive weeks. $G_{1}$ flies were

682 then allowed to mate inter se and eggs were collected in the same manner. $\mathrm{G}_{2}$ pupae were

683 then analyzed phenotypically and separated according to color of pupae (brown, mosaic or 684 white).

685 For C. capitata CRISPR/Cas9 gene editing, lyophilized Cas9 protein (PNA Bio Inc, CP01) was reconstituted to a stock concentration of $1 \mu \mathrm{g} / \mu \mathrm{l}$ in $20 \mathrm{mM}$ Hepes, $150 \mathrm{mM} \mathrm{KCl}, 2 \%$ sucrose and $1 \mathrm{mM}$ DTT ( $\mathrm{pH} 7.5$ ) and stored at $-80^{\circ} \mathrm{C}$ until use. A guide RNA (gRNA_MFS), targeting the third CDS exon of CCMFS was designed and tested for potential off target effects using Geneious Prime ${ }^{52}$ and the C. capitata genome annotation Ccap2.1. In silico target site analysis predicted an on-target activity score of 0.615 (scores are between 0 and 1 ; the higher the score the higher the expected activity ${ }^{66}$ and zero off-targets sites in the medfly genome. Guide RNA was synthesized by in vitro transcription of linear double-stranded DNA template as previously described ${ }^{67}$ using primers $P_{\_} 1753$ and P_369 (Supplementary Table 4), Q5 HF polymerase, and a Bio-Rad C1000 Touch thermal cycler $\left[98^{\circ} \mathrm{C}, 30 \mathrm{~s} ; 35\right.$ cycles of $\left(98^{\circ} \mathrm{C}, 10\right.$ $\left.\mathrm{s} ; 58^{\circ} \mathrm{C}, 20 \mathrm{~s} ; 72^{\circ} \mathrm{C}, 20 \mathrm{~s}\right) ; 72^{\circ} \mathrm{C}, 2 \mathrm{~min}$ ] and HiScribe ${ }^{\mathrm{TM}} \mathrm{T} 7$ High Yield RNA Synthesis. Injection mixes for microinjection of embryos contained $360 \mathrm{ng} / \mu \mathrm{l}$ Cas 9 protein $(1 \mu \mathrm{g} / \mu \mathrm{l}$, dissolved in its formulation buffer), $200 \mathrm{ng} / \mu \mathrm{l}$ gRNA_MFS and an end-concentration of $300 \mathrm{mM} \mathrm{KCl}$ according to previous studies ${ }^{67,68}$. The mix was freshly prepared on ice followed by an incubation step for 10 min at $37^{\circ} \mathrm{C}$ to allow pre-assembly of gRNA-Cas9 ribonucleoprotein complexes and stored on ice prior to injections. Microinjections were conducted in wild type Egll C. capitata embryos. Eggs were collected over a 30-40 min time period and prepared for injection as previously described ${ }^{67}$. Injections were performed using siliconized quartz glass needles based micropipette puller. The injection station consisted of a manual micromanipulator (MNmicroscope (SDF PLAPO 1xPF objective). The microscope slide with the injected embryos 
was placed in a Petri dish containing moist tissue paper in an oxygen chamber (max. 2 psi). Hatched first instar larvae were transferred from the oil to larval food.

As complementation assay, reciprocal crosses between surviving $\mathrm{G}_{0}$ adults and virgin white pupae strain \#1402_22m1B (pBac_fa_attP-TREhs43-Cctra-I-hid ${ }^{A l a 5}$ _SV40_a_PUb-n/s-EGFP$S V 40)\left(w p^{- \text {(nat) }}\right)^{24}$ were set up either single paired (six cages) or in groups of seven to ten flies (seven cages). Eggs were collected three times every 1-2 days. Progeny $\left(\mathrm{G}_{1}\right)$ exhibiting the white pupae phenotype ( $w p^{-(\text {nat)-(CRISPR) }}$ ) were assayed via non-lethal genotyping and sorted according to mutation genotype (see Fig. 4). Genotypes 'A-H' were group-backcrossed into WT Egll $\left(w p^{+++}\right)$, genotype 'C' siblings massed crossed. Eggs were collected 4 times every 12 days. Generation $\mathrm{G}_{2}$ flies were analyzed via PCR using three primers, specific for $w p^{+}$and $w p^{- \text {(CRISPR) }}$ or $w p^{- \text {(nat) }}$ allele size respectively. Offspring of outcross cages showed brown pupae phenotype and either $w p^{+- \text {(nat) }}$ or $w p^{+ \text {- (CRISPR) }}$ genotype. In order to make mutations $A, D$, and $\mathrm{H}$ homozygous, 40 flies (25 females, 15 males) were genotyped each, and $w p^{+ \text {-(CRISPR) }}$ positive flies were inbred (mutation A: 15 females, 7 males, mutation D: 12 females, 7 males, mutation $\mathrm{H}$ : 11 females, 8 males). $\mathrm{G}_{3}$ showing white pupae phenotype was homozygous for $w p^{-(\mathrm{CRISPR})}$ mutations $A, D$, or $\mathrm{H}$, respectively and was used to establish lines. Inbreeding of mutation $\mathrm{C}$ $w p^{-(\text {nat)|-(CRISPR) }}$ flies produced only white pupae offspring, based on either $w p^{- \text {(nat)|-(nat) }}, w p^{-(\text {nat) })-}$ ${ }^{(C R I S P R)}$ or $w p^{-(C R I S P R)-(C R I S P R)} .94$ flies (46 females, 48 males) were genotyped, homozygous $w p^{-}$ (CRISPR) were inbred to establish a line (13 females, 8 males).

Molecular analyses of wp mutants and mosaics. In B. tryoni, genomic DNA was isolated for genotyping from $\mathrm{G}_{2}$ pupae using the DNeasy Blood and Tissue Kit (Qiagen). PCR amplicons spanning both BtMFS guide recognition sites were generated using Q5 polymerase (NEB) with primers BtMFS_5primeF and BtMFS_exon2R (Supplementary Table 4). Products were purified using MinElute PCR Purification Kit (Qiagen), ligated into pGEM-t-easy vector (Promega) and transformed into DH5a cells. Plasmids were purified with Wizard Plus SV Minipreps (Promega) and sequenced.

In C. capitata, non-lethal genotyping was performed to identify parental genotypes before setting up crosses. Therefore, genomic DNA was extracted from single legs of $G_{1}$ and $G_{2}$ flies following a protocol established by ${ }^{69}$. Single legs of anesthetized flies were cut at the proximal femur using scissors. Legs were then homogenized by ceramic beads and $50 \mu$ buffer (10 $\mathrm{mM}$ Tris- $\mathrm{Cl}, \mathrm{pH}$ 8.2, $1 \mathrm{mM}$ EDTA, $25 \mathrm{mM} \mathrm{NaCl})$ for $15 \mathrm{~s}(6 \mathrm{~m} / \mathrm{s})$ using a FastPrep-24 ${ }^{\text {TM }} 5 \mathrm{G}$ homogenizer. Then, $30 \mu \mathrm{l}$ buffer and $1.7 \mu$ proteinase-K $(2.5 \mathrm{U} / \mathrm{mg})$ were added, incubated for $1 \mathrm{~h}$ at $37^{\circ} \mathrm{C}$ and the reaction stopped by $4 \mathrm{~min}$ at $98^{\circ} \mathrm{C}$. The reaction mix was cooled down on ice and used for PCR. For $\mathrm{G}_{1}$ flies, $\mathrm{PCR}$ on wp was performed in a $25 \mu$ reaction volume using the DreamTaq polymerase, primer pair P_1643 and P_1644 (Supplementary Table 4), and $3.75 \mu$ l reaction mix. Different amplicon sizes are expected for brown ( $w p^{+}$and $\left.w p^{-(C R I S P R)}\right)$ 
744 and white pupae $\left(w p^{- \text {(nat) }}\right)$ alleles, $724 \mathrm{bp}$ and 8,872 bp in size, respectively. The $w p^{- \text {(nat) }}$

745 amplicon was excluded via PCR settings $\left[95^{\circ} \mathrm{C}, 3 \mathrm{~min} ; 35\right.$ cycles of $\left(95^{\circ} \mathrm{C}, 30 \mathrm{~s} ; 56^{\circ} \mathrm{C}, 30 \mathrm{~s}\right.$;

$\left.74672^{\circ} \mathrm{C}, 1 \mathrm{~min}\right) ; 72^{\circ} \mathrm{C}, 5 \mathrm{~min}$ ]. The $724 \mathrm{bp} \mathrm{PCR}$ product was verified by agarose electrophoresis

747 and purified from the PCR reaction using the DNA Clean \& Concentrator ${ }^{\mathrm{TM}}-5$ kit. PCR products

748 were sequenced using primer $P_{-} 1644$ and sequences analyzed using the Geneious Prime

749 Software Package (6). In generation $\mathrm{G}_{2}$, flies were analyzed using multiplex PCR with primers

750 P_1657, $P_{-}$1643, and P_1644 (Supplementary Table 4), to distinguish between the $w p^{- \text {(nat) }}$

751 (457 bp; $P_{-} 1643$ and $P_{-}$1657), and $w p^{-(\text {CRISPR) }}$ alleles (724 bp; $P_{-} 1643$ and $\left.P 1644\right)$ using the

752 PCR protocol $\left[95^{\circ} \mathrm{C}, 3 \mathrm{~min} ; 35\right.$ cycles of $\left.\left.\left(95^{\circ} \mathrm{C}, 30 \mathrm{~s} ; 56^{\circ} \mathrm{C}, 30 \mathrm{~s} ; 72^{\circ} \mathrm{C}, 1 \mathrm{~min}\right) ; 72^{\circ} \mathrm{C}, 5 \mathrm{~min}\right]\right)$.

754 Image acquisition. Images of $B$. tryoni pupae were taken with an Olympus SZXI6

755 microscope, Olympus DP74 camera and Olympus LF-PS2 light source using the Olympus

756 stream basic 2.3.3 software. Images of $C$. capitata pupae were taken with a Keyence digital

757 microscope VHX-5000. Image processing was conducted with Adobe Photoshop CS5.1

758 software to apply moderate changes to image brightness and contrast. Changes were applied

759 equally across the entire image and throughout all images. 
Not applicable

762

\section{Consent for publication}

763

Not applicable

764

765

766

767

768

769

770

771

772

773

774

775

776

777

778

779

780

\section{Availability of data and material}

All data generated or analyzed are included in this article.

\section{Funding}

This study was financially supported by the Joint FAO/IAEA Insect Pest Control Subprogramme of the Joint FAO/IAEA Division of Nuclear Techniques in Food and Agriculture. The project has also been funded by the Horticulture Innovation Australia (FF17000, FF18002), using funds from the Australian Government, and co-investment from Macquarie University and South Australian Research and Development Institute (SARDI). SWB was supported by the Australian Research Council (FT140101303) and Hermon Slade Foundation grant HSF 18/6. Furthermore, this work was supported by the Emmy Noether program of the German Research Foundation (SCHE 1833/1-1; to MFS) and the LOEWE Center for Insect Biotechnology and Bioresources of the Hessen State Ministry for Higher Education, Research and the Arts (HMWK; to MFS). The work was also supported by Canadian Foundation for Innovation and Genome Canada Genome Technology Platform awards and the International Atomic Energy Agency research contact no. 23358 as part of the Coordinated Research Project "Generic approach for the development of genetic sexing strains for SIT applications" (JR).

\section{Acknowledgements}

This study was benefitted from discussions at International Atomic Energy Agency funded meetings for the Coordinated Research Projects (CRPs) and particularly the CRP on "Generic approach for the development of genetic sexing strains for SIT applications". The authors also wish to thank Tanja Rehling, Jakob Martin and Johanna Rühl for technical assistance and Germano Sollazzo for helping with injections and primers design (Justus-Liebig University Gießen and Insect Pest Control Laboratory); Elena Isabel Cancio Martinez, Thilakasiri Dammalage, Sohel Ahmad and Gülizar Pillwax for insect rearing (Insect Pest Control Laboratory), Shu-Huang Chen (McGill University) for technical assistance with Nanopore library preparations; and Arjen van't Hof (University of Liverpool) for constructing libraries from micro-dissected chromosomes.

\section{Competing Interests Statement}

The authors declare no competing interests. 


\section{References}

796 1. Robinson, A.S. \& Hooper, G. Fruit flies: their biology, natural enemies, and control, Vol. 1. (Elsevier, Amsterdam; New York; 1989).

2. Suckling, D.M. et al. Eradication of tephritid fruit fly pest populations: outcomes and prospects. Pest Manag Sci 72, 456-465 (2016).

800 Dyck, V.A. et al. in Sterile insect technique - principles and practice in area-wide integrated pest management. (eds. V.A. Dyck, J. Hendrichs \& A.S. Robinson) 525-545

802 (Springer, Dordrecht, NL; 2005).

803

804

4. Vreysen, M., Robinson, A.S. \& Hendrichs, J. Area-wide control of insect pests: from research to field implementation. (Springer, Dordrecht, NL; 2007).

805

5. Rendon, P., Mclnnis, D., Lance, D. \& Stewart, J. Medfly (Diptera: Tephritidae) genetic sexing: large-scale field comparison of males-only and bisexual sterile fly releases in

806

807 Guatemala. Journal of Economic Entomology 97, 1547-1553 (2004).

808

6. Franz, G. in Sterile insect technique - principles and practice in area-wide integrated

809

810

811 pest management. (eds. V.A. Dyck, J. Hendrichs \& A.S. Robinson) 427-451 (Springer, Dordrecht, NL; 2005).

7. Augustinos, A.A. et al. Ceratitis capitata genetic sexing strains: laboratory evaluation of strains from mass-rearing facilities worldwide. Entomologia Experimentalis et Applicata 164, 305-317 (2017).

814

8. Zacharopoulou, A. et al. A review of more than 30 years of cytogenetic studies of Tephritidae in support of sterile insect technique and global trade. Entomologia Experimentalis et Applicata 164, 204-225 (2017).

818

9. Rössler, Y. The genetics of the Mediterranean fruit fly: a "white pupae" Mutant. Annals of the Entomological Society of America 72, 583-585 (1979).

820

10. Rössler, Y. \& Koltin, Y. The genetics of the Mediterranean fruit fly, Ceratitis capitata: three morphological mutations. Annals of the Entomological Society of America 69, 604-608 (1976).

11. McCombs, S.D. \& Saul, S.H. Linkage analysis of five new genetic markers of the oriental fruit fly, Bactrocera dorsalis (Diptera: Tephritidae). The Journal of heredity 83, 199-203 (1992).

12. Mclnnis, D.O. et al. Development of a pupal color-based genetic sexing strain of the melon fly, Bactrocera cucurbitae (Coquillett) (Diptera: Tephritidae). Annals of the Entomological Society of America 97, 1026-1033 (2004).

13. Wappner, P. et al. White pupa: A Ceratitis capitata mutant lacking catecholamines for tanning the puparium, Vol. 25. (1995).

14. Rössler, Y. \& Rosenthal, H. Genetics of the Mediterranean fruit fly (Diptera: Tephritidae): morphological mutants on chromosome five. Annals of the Entomological Society of America 85, 525-531 (1992).

15. Kerremans, P. \& Franz, G. Cytogenetic analysis of chromosome 5 from the Mediterranean fruit fly, Ceratitis capitata. Chromosoma 103, 142-146 (1994).

16. Papanicolaou, A. et al. The whole genome sequence of the Mediterranean fruit fly, Ceratitis capitata (Wiedemann), reveals insights into the biology and adaptive evolution of a highly invasive pest species. Genome Biology 17, 192 (2016).

838 17. Papathanos, P.A. et al. A perspective on the need and current status of efficient sex 839 separation methods for mosquito genetic control. Parasites \& Vectors 11, 654 (2018). 
18. Sim, S.B. \& Geib, S.M. A chromosome-scale assembly of the Bactrocera cucurbitae genome provides insight to the genetic basis of white pupae. G3 (Bethesda, Md.) 7 , 1927-1940 (2017).

19. Sim, S.B., Ruiz-Arce, R., Barr, N.B. \& Geib, S.M. A new diagnostic resource for Ceratitis capitata strain identification based on QTL mapping. G3 (Bethesda, Md.) 7, 3637-3647 (2017).

20. Zdobnov, E.M. et al. OrthoDB v9.1: cataloging evolutionary and functional annotations for animal, fungal, plant, archaeal, bacterial and viral orthologs. Nucleic Acids Res 45 , D744-D749 (2017).

21. San Jose, M. et al. Incongruence between molecules and morphology: A seven-gene phylogeny of Dacini fruit flies paves the way for reclassification (Diptera: Tephritidae). Mol Phylogenet Evol 121, 139-149 (2018).

22. Choo, A., Crisp, P., Saint, R., O'Keefe, L.V. \& Baxter, S.W. CRISPR/Cas9-mediated mutagenesis of the white gene in the tephritid pest Bactrocera tryoni. Journal of Applied Entomology 142, 52-58 (2018).

23. Gariou-Papalexiou, A. et al. Polytene chromosomes as tools in the genetic analysis of the Mediterranean fruit fly, Ceratitis capitata. Genetica 116, 59-71 (2002).

24. Ogaugwu, C.E., Schetelig, M.F. \& Wimmer, E.A. Transgenic sexing system for Ceratitis capitata (Diptera: Tephritidae) based on female-specific embryonic lethality. Insect Biochemistry and Molecular Biology 43, 1-8 (2013).

25. Davis, A.W. et al. Rescue of hybrid sterility in crosses between $D$. melanogaster and D. simulans. Nature 380, 157-159 (1996).

26. Araripe, L.O., Montenegro, H., Lemos, B. \& Hartl, D.L. Fine-scale genetic mapping of a hybrid sterility factor between Drosophila simulans and $D$. mauritiana: the varied and elusive functions of "speciation genes". BMC Evol Biol 10, 385 (2010).

27. Brideau, N.J. \& Barbash, D.A. Functional conservation of the Drosophila hybrid incompatibility gene Lhr. BMC Evol Biol 11, 57 (2011).

28. Kotov, A.A. et al. piRNA silencing contributes to interspecies hybrid sterility and reproductive isolation in Drosophila melanogaster. Nucleic Acids Res 47, 4255-4271 (2019).

29. Barbash, D.A. Ninety years of Drosophila melanogaster hybrids. Genetics 186, 1-8 (2010).

30. Bedo, D.G. \& Zacharopoulou, A. Inter-tissue variability of polytene chromosome banding patterns. Trends Genet 4, 90-91 (1988).

31. Kriventseva, E.V. et al. OrthoDB v10: sampling the diversity of animal, plant, fungal, protist, bacterial and viral genomes for evolutionary and functional annotations of orthologs. Nucleic Acids Res 47, D807-d811 (2019).

32. Zhao, Y. et al. A major facilitator superfamily protein participates in the reddish brown pigmentation in Bombyx mori. J Insect Physiol 58, 1397-1405 (2012).

33. The modEncode Consortium et al. Identification of functional elements and regulatory circuits by Drosophila modENCODE. Science (New York, N.Y.) 330, 1787-1797 (2010).

34. Wright, T.R. The genetics of biogenic amine metabolism, sclerotization, and melanization in Drosophila melanogaster. Adv Genet 24, 127-222 (1987).

35. Bourtzis, K., Psachoulia, C. \& Marmaras, V.J. Evidence that different integumental phosphatases exist during development in the Mediterranean fruit fly Ceratitis capitata: possible involvement in pupariation. Comparative Biochemistry and Physiology Part B: Comparative Biochemistry 98, 411-416 (1991). 
36. Meccariello, A. et al. Maleness-on-the- $Y(M o Y)$ orchestrates male sex determination in major agricultural fruit fly pests. Science $365,1457-1460$ (2019).

37. Hall, A.B. et al. Sex determination. A male-determining factor in the mosquito Aedes aegypti. Science 348, 1268-1270 (2015).

38. Liu, P. et al. Nix is a male-determining factor in the Asian tiger mosquito Aedes albopictus. Insect Biochem Mol Biol 118, 103311 (2019).

39. Ward, C.M., To, T.H. \& Pederson, S.M. ngsReports: a Bioconductor package for managing FastQC reports and other NGS related log files. Bioinformatics 36, 25872588 (2020).

40. Sedlazeck, F.J., Rescheneder, P. \& von Haeseler, A. NextGenMap: fast and accurate read mapping in highly polymorphic genomes. Bioinformatics 29, 2790-2791 (2013).

41. Garrison, E. \& Marth, G. in arXiv e-prints (2012).

42. $\mathrm{Li}, \mathrm{H}$. A statistical framework for SNP calling, mutation discovery, association mapping and population genetical parameter estimation from sequencing data. Bioinformatics 27, 2987-2993 (2011).

43. Zheng, $X$. et al. SeqArray-a storage-efficient high-performance data format for WGS variant calls. Bioinformatics 33, 2251-2257 (2017).

44. Simão, F.A., Waterhouse, R.M., loannidis, P., Kriventseva, E.V. \& Zdobnov, E.M. BUSCO: assessing genome assembly and annotation completeness with single-copy orthologs. Bioinformatics 31, 3210-3212 (2015).

45. Waterhouse, R.M. et al. BUSCO applications from quality assessments to gene prediction and phylogenomics. Mol Biol Evol 35, 543-548 (2018).

46. Stamatakis, A. RAxML version 8: a tool for phylogenetic analysis and post-analysis of large phylogenies. Bioinformatics 30, 1312-1313 (2014).

47. Zhang, C., Rabiee, M., Sayyari, E. \& Mirarab, S. ASTRAL-III: polynomial time species tree reconstruction from partially resolved gene trees. BMC Bioinformatics 19, 153 (2018).

48. Martin, S.H. \& Van Belleghem, S.M. Exploring evolutionary relationships across the genome using topology weighting. Genetics 206, 429-438 (2017).

49. Finn, R.D., Clements, J. \& Eddy, S.R. HMMER web server: interactive sequence similarity searching. Nucleic Acids Res 39, W29-37 (2011).

50. $\mathrm{Li}, \mathrm{H}$. et al. The Sequence Alignment/Map format and SAMtools. Bioinformatics 25, 2078-2079 (2009).

51. Zimin, A.V. et al. The MaSuRCA genome assembler. Bioinformatics 29, 2669-2677 (2013).

52. Kearse, M. et al. Geneious Basic: an integrated and extendable desktop software platform for the organization and analysis of sequence data. Bioinformatics 28, 1647 1649 (2012).

53. Green, M.R. \& Sambrook, J. Isolation of High-Molecular-Weight DNA using organic solvents. Cold Spring Harb Protoc 2017, pdb.prot093450 (2017).

54. Koren, S. et al. Canu: scalable and accurate long-read assembly via adaptive k-mer weighting and repeat separation. Genome Res 27, 722-736 (2017).

55. Huang, S. et al. HaploMerger: reconstructing allelic relationships for polymorphic diploid genome assemblies. Genome Res 22, 1581-1588 (2012).

56. Ghurye, J., Pop, M., Koren, S., Bickhart, D. \& Chin, C.S. Scaffolding of long read assemblies using long range contact information. BMC Genomics 18, 527 (2017).

57. Haas, B.J. et al. De novo transcript sequence reconstruction from RNA-seq using the Trinity platform for reference generation and analysis. Nat Protoc 8, 1494-1512 (2013). 
58. Li, H. Minimap2: pairwise alignment for nucleotide sequences. Bioinformatics 34, 3094-3100 (2018).

59. Dobin, A. et al. STAR: ultrafast universal RNA-seq aligner. Bioinformatics 29, 15-21 (2013).

60. Robinson, J.T. et al. Integrative genomics viewer. Nature biotechnology 29, 24-26 (2011).

61. Zacharopoulou, A. \& Franz, G. Genetic and cytogenetic characterization of genetic sexing strains of Bactrocera dorsalis and Bactrocera cucurbitae (Diptera: Tephritidae). $J$ Econ Entomol 106, 995-1003 (2013).

62. Zacharopoulou, A. et al. The genome of the Mediterranean fruit fly Ceratitis capitata: localization of molecular markers by in situ hybridization to salivary gland polytene chromosomes. Chromosoma 101, 448-455 (1992).

63. Mavragani-Tsipidou, P. et al. in Protocols for cytogenetic mapping of arthropod genomes. (ed. I. Sakharov) 1-62 (CRC Press, Taylor and Francis Group, LLC, Florida, USA 2014).

64. Zacharopoulou, A. Polytene chromosome maps in the medfly Ceratitis capitata Genome 33, 184-197 (1990).

65. Concordet, J.P. \& Haeussler, M. CRISPOR: intuitive guide selection for CRISPR/Cas9 genome editing experiments and screens. Nucleic Acids Res 46, W242-W245 (2018).

66. Doench, J.G. et al. Rational design of highly active sgRNAs for CRISPR-Cas9mediated gene inactivation. Nature biotechnology 32, 1262-1267 (2014).

67. Aumann, R.A., Schetelig, M.F. \& Häcker, I. Highly efficient genome editing by homology-directed repair using Cas9 protein in Ceratitis capitata. Insect Biochemistry and Molecular Biology 101, 85-93 (2018).

68. Burger, A. et al. Maximizing mutagenesis with solubilized CRISPR-Cas9 ribonucleoprotein complexes. Development (Cambridge, England) 143, 2025-2037 (2016).

69. Carvalho, G.B., Ja, W.W. \& Benzer, S. Non-lethal PCR genotyping of single Drosophila. Biotechniques 46, 312-314 (2009). 